\title{
Chitosan oligosaccharides packaged into rat adipose mesenchymal stem cells-derived extracellular vesicles facilitating cartilage injury repair and alleviating osteoarthritis
}

Shenglong $\mathrm{Li}^{1,2+}{ }^{\circ}$, Jie Liu ${ }^{3 \dagger}$, Siyu Liu ${ }^{1}$, Weijie Jiao ${ }^{1}$ and Xiaohong Wang ${ }^{1,4^{*}}$

\begin{abstract}
Objectives: This study aimed to investigate the roles of adipose mesenchymal stem cell (AMSC)-derived extracellular vesicles (EVs) binding with chitosan oligosaccharides (COS) in cartilage injury, as well as the related mechanisms.

Results: IL-1 $\beta$ treatment significantly inhibited the viability and migration of chondrocytes and enhanced cell apoptosis $(P<0.05)$, while chitosan oligosaccharides and extracellular vesicles-chitosan oligosaccharide conjugates (EVsCOS/EVs-COS conjugates) reversed the changes induced by IL-1 $\beta(P<0.05)$, and the effects of extracellular vesicleschitosan oligosaccharide conjugates were better than those of chitosan oligosaccharides $(P<0.05)$. After cartilage damage, IL-1ß, OPN, and p53 were significantly upregulated, COL1A1, COL2A1, OCN, RUNX2, p-Akt/Akt, PI3K, c-Myc, and $\mathrm{BCl} 2$ were markedly downregulated, and extracellular vesicles-chitosan oligosaccharide conjugates reversed the expression induced by cartilage injury. Through sequencing, 760 differentially expressed genes (DEGs) clustered into four expression patterns were associated with negative regulation of the canonical Wnt, PI3K-Akt, AMPK, and MAPK signaling pathways.
\end{abstract}

Conclusion: Extracellular vesicles-chitosan oligosaccharide conjugates may serve as a new cell-free biomaterial to facilitate cartilage injury repair and improve osteoarthritis.

Keywords: EVs, Chitosan oligosaccharides, EVs-COS conjugates, Cartilage injury repair, Osteoarthritis

\footnotetext{
*Correspondence: wangxiaohong709@163.com; wangxiaohong@mail.

tsinghua.edu.cn

'Shenglong Li and Jie Liu contributed equally to this work

${ }^{1}$ Department of Tissue Engineering, Center of 3D Printing \& Organ

Manufacturing, School of Intelligent Medicine, China Medical University

(CMU), No. 77 Puhe Road, Shenyang North New Area, Shenyang 110122,

China

Full list of author information is available at the end of the article
}

(c) The Author(s) 2021. Open Access This article is licensed under a Creative Commons Attribution 4.0 International License, which permits use, sharing, adaptation, distribution and reproduction in any medium or format, as long as you give appropriate credit to the original author(s) and the source, provide a link to the Creative Commons licence, and indicate if changes were made. The images or other third party material in this article are included in the article's Creative Commons licence, unless indicated otherwise in a credit line to the material. If material is not included in the article's Creative Commons licence and your intended use is not permitted by statutory regulation or exceeds the permitted use, you will need to obtain permission directly from the copyright holder. To view a copy of this licence, visit http://creativecommons.org/licenses/by/4.0/. The Creative Commons Public Domain Dedication waiver (http://creativeco mmons.org/publicdomain/zero/1.0/) applies to the data made available in this article, unless otherwise stated in a credit line to the data. 


\section{Graphical Abstract}

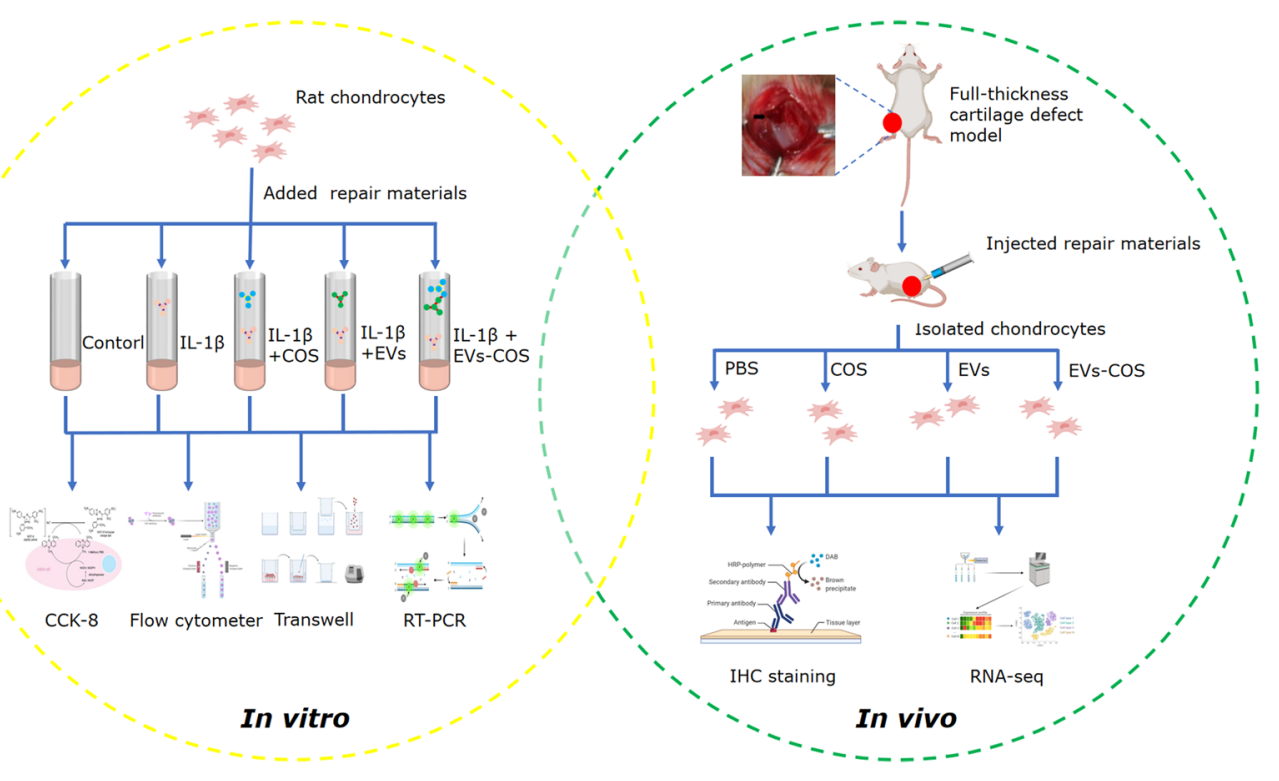

\section{Background}

Articular cartilage, a non-self-repairing tissue, is mainly composed of water, proteoglycan, and collagen, which together determine the functional characteristics of cartilage tissues [1]. Cartilage injury usually marks the occurrence of tissue degeneration, progressive deterioration, subchondral osteosclerosis, and osteoarthritis (OA) [2]. OA clinically manifests as slow progression of joint pain, tenderness, stiffness, joint swelling, limited movement, and joint deformity [3], and is a major cause of disability, affecting approximately 240 million people globally [4]. Currently, drugs used to alleviate the symptoms of OA include steroid injections, non-steroidal anti-inflammatory drugs (NSAIDs), and opioids [5, 6]. However, the long-term use of these drugs may result in side effects, such as gastrointestinal, renal, and cardiovascular diseases [7]. Nanocomposites, including organic-inorganic, inorganic-inorganic, and bioinorganic nanomaterials, have been reported in bone tissue regeneration engineering, such as hydroxyapatite (HA) with chitosan, polycaprolactone/bioglass, and HA-gelatin nanocomposites [8]. By combining nanotechnology-based drug delivery systems, the bioavailability, pharmacokinetics and pharmacodynamics of drugs in bone tissue can be improved, thus improving therapeutic efficacy while reducing side effects. Owing to the immunogenicity of the receptor cells, rapid blood clearance, cytotoxicity, and poor biological distribution of these nanocomposites, their use has been limited [9]. Therefore, more therapeutic strategies are urgently needed to improve cartilage injury repair and manage OA.

Mesenchymal stem cells (MSCs), which can be isolated from many adult organs, are self-renewing multipotent progenitors, and can differentiate into a variety of cell lineages, such as adipocytes, osteoblasts, and chondrocytes [10]. Increasing evidence has shown that MSC transplantation promotes tissue regeneration, including fracture, wound healing, and cartilage repair [11]. A previous study indicated that bone marrow MSCs could enhance articular cartilage repair and regeneration, as well as improve the quality of life of knee OA [12]. In addition, owing to their relatively easy isolation, high yield, and strong potential for proliferation and differentiation [13], adipose MSCs (AMSCs) have been widely used in various biomedical applications. A previous double-blinded clinical trial showed that intraarticular injection of AMSCs could improve cartilage defects and relieve pain in knee OA patients, without causing adverse events at 6 months' follow-up [14]. Another study reported that AMSCs with BMP9 overexpression promoted cartilage repair and differentiation through the Notch1/Jagged1 signaling pathway [15]. These findings suggest that AMSCs can promote cartilage injury repair and improve OA. Nevertheless, the clinical effects of traditional AMSC transplantation methods have been greatly limited by their stability, safety, and immune-mediated rejection.

Extracellular vesicles (EVs) are released by a variety of cells [16], and can serve as a tool for cell-to-cell communication. They can selectively encapsulate protein 
molecules, genes (RNA and DNA), cytokines, and other functional bioactive substances derived from the cells and deliver them to the extracellular environment or other target cells [17]. EVs derived from AMSCs have been considered an important part of cell-free regenerative medicine because they carry special bioactive substances and possess the distinctive and typical characteristics of AMSCs [18]. Many studies have confirmed that AMSC-derived EVs play essential roles in skin healing and regeneration, neurodegenerative diseases, ischemia reperfusion injury, and obesity [19]. A recent study demonstrated that AMSC-derived exosomal microRNA (miR)-19b could enhance the ability to heal wounds by targeting the CCL1/TGF- $\beta$ signaling axis [20]. Another study also reported that AMSC-derived EVs promoted cartilage formation by upregulating miR145 and miR-221, and inhibited inflammation by downregulating pro-inflammatory markers (IL-6, NF-kB, and TNF- $\alpha$ ) [21]. In addition, EVs have better targeting ability and safety and are attracting increasing attention. Therefore, AMSC-derived EVs are expected to be a new clinical strategy for cartilage injury repair and OA treatment.

Chitosan, a natural source of polysaccharides, has been widely used in biomedical fields, such as tissue engineering and pharmaceutical preparation, owing to its biodegradable, biocompatible, and non-toxic properties [22]. Previous studies have shown that chitosan has antibacterial and anticoagulant properties, can promote wound healing, and can improve immune functions [23-25]. Carboxymethyl chitosan combined with hydroxyapatite has been reported to enhance alkaline phosphatase (ALP) activity and promote osteoblast differentiation and new bone formation/maturation in vivo and in vitro, and may therefore, be used as a promising scaffold for bone tissue engineering [26]. Chitosan/poly (vinyl alcohol) could allow AMSCs to proliferate and differentiate into chondrocytes, improving their clinical effects on OA pathology. A previous study used AMSC-derived exosomes to immobilize the polydopamine-coated PLGA (PLGA/pDA) scaffolds, and found that exosomes could be released from PLGA/pDA scaffolds and significantly promote bone formation and regeneration of bone tissue [27]. Therefore, we speculate that chitosan can be used as a scaffold to promote the effects of AMSC-derived EVs. However, the roles of chitosan combined with AMSCderived EVs in cartilage injury repair and the underlying mechanisms remain unclear.
In this study, both in vitro and in vivo rat cartilage injury models were constructed to systematically investigate the effects and related mechanisms of low molecular chitosan oligosaccharides (COS) packaged into rat AMSC-derived EVs in cartilage injury. These findings provide new insights and strategies for drug development for the treatment of $\mathrm{OA}$ and other degenerative joint diseases based on cartilage injury.

\section{Results \\ Determination of the optimal concentrations of EVs and COS}

To determine the optimal concentrations of EVs and COS for further experiments, chondrocytes were treated with different concentrations of EVs and COS for $48 \mathrm{~h}$, after which their viability was determined. As shown in Additional file 1: Figure S1 A, cell viability after exposure to $5,10,20$, and $50 \mu \mathrm{g} / \mathrm{mL}$ EV treatment was significantly increased compared with the untreated cells $(P<0.05)$, and the cell viability was the highest in the cells treated with $20 \mu \mathrm{g} / \mathrm{mL}$ EVs (Additional file 1: Figure S1A). Furthermore, there was no significant difference in cell viability among the cells treated with 0,10 , and $20 \mu \mathrm{g} / \mathrm{mL}$ COS $(P>0.05$, Additional file 1: Figure S1B). When the cells were treated with $40,80,160,320,640$, and $1280 \mu \mathrm{g} /$ $\mathrm{mL} C O S$, their viability was significantly enhanced $(P<0.05)$, and the viability of the cells treated with $320 \mu \mathrm{g} / \mathrm{mL}$ COS was the highest (Additional file 1: Figure $\mathrm{S} 1 \mathrm{~B})$. Based on these results, $20 \mu \mathrm{g} / \mathrm{mL}$ EVs and $320 \mu \mathrm{g} /$ $\mathrm{mL} C O S$ were selected for subsequent experiments.

\section{Identification of rat AMSCs-derived EVs, EVs-COS, and rat chondrocytes}

EVs isolated from rat AMSCs were characterized using TEM, NTA, and Western blotting. TEM showed that the extracted substances were cup-shaped or nearly round with a diameter of approximately $100 \mathrm{~nm}$ (Fig. 1A). The results of NTA showed that the major peak of isolated substances was approximately $120 \mathrm{~nm}$, and that the average peak was $133.9 \pm 0.8 \mathrm{~nm}$ (Fig. 1B), which was in accordance with the previously reported size distribution of EVs [28]. In addition, Western blot results demonstrated that CD3, TSG101, and CD9, which are EV markers, were all expressed in the EVs, whereas calnexin was only expressed in the cells (Fig. 1C). These results indicate that EVs were successfully isolated from rat AMSCs.

(See figure on next page.)

Fig. 1 Characterization of extracellular vesicles (EVs) isolated from rat adipose mesenchymal stem cells (AMSCs), and EVs-chitosan oligosaccharide (COS) conjugates (EVs-COS). A The morphology of rat AMSCs-derived EVs observed by transmission electron microscopy (TEM). B The particle size distribution of EVs determined by a Nanosight NS300 particle size analyzer (NTA). C The expressions of EVs surface markers (CD63, TSG101, and CD9) measured using Western blot. D The morphology of EVs-COS by using TEM. E The particle size distribution of EVs-COS measured by NTA. F EVs and EVs-COS taken up by rat chondrocytes visualized using PKH67 staining kit (green fluorescence) 

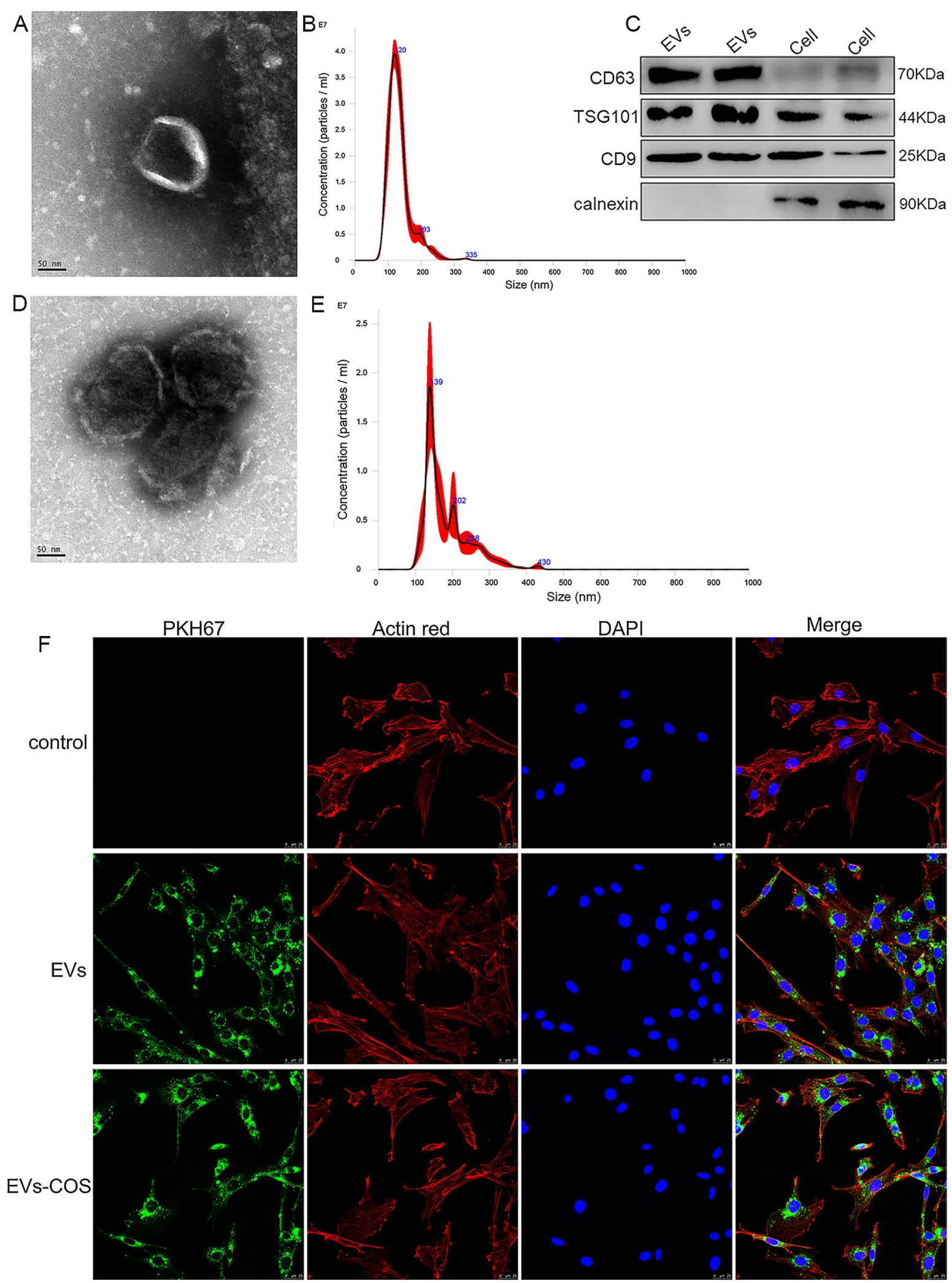

Fig. 1 (See legend on previous page.) 
After COS bound with EVs, TEM and NTA were used to observe the morphology and determine the size of the extracellular vesicles-chitosan oligosaccharide conjugates (EVs-COS), respectively. The morphology of EVs-COS was nearly round or round with a diameter of approximately $100 \mathrm{~nm}$ (Fig. 1D), which is consistent with the characteristics of EVs. NTA results showed that the major peak of EVs-COS was $139 \mathrm{~nm}$, and that the average peak was $183.4 \pm 1.5 \mathrm{~nm}$ (Fig. 1E), which indicated that the size of EVs-COS was higher than that of EVs. The TEM and NTA results confirmed that COS successfully bound to EVs.

In addition, chondrocytes isolated from the cartilage tissues of rats were identified by determining the expression of collagen II. Additional file 2: Figure S2 shows that the cells were stained dark yellow-brown, a typical characteristic of isolated chondrocytes. Positive results were denoted by the staining of the collagen II components.

\section{EVs and EVs-COS uptake by chondrocytes}

PKH67 was used to label EVs and EVs-COS (green fluorescence), and then the labeled EVs and EV-COS were co-cultured with rat chondrocytes. After co-culturing for $48 \mathrm{~h}$, the cytoskeleton of chondrocytes was stained with actin red (red fluorescence). It was observed that most chondrocytes displayed intracellular green fluorescence (Fig. 1F), indicating that EVs isolated from rat AMSCs and EVs-COS could be taken up by rat chondrocytes.

\section{Effects of EVs-COS on cell viability and apoptosis of chondrocytes}

To understand the effects of EVs-COS on cartilage injury repair, IL-1 $\beta$ was used to establish a chondrocyte injury model. After culturing for 24,48 , and $72 \mathrm{~h}$, cell viability and apoptosis were measured. Cell viability in the IL- $1 \beta$ group was significantly inhibited compared with that in the control group $(P<0.05$, Fig. $2 A)$. No significant difference in cell viability was found between the IL- $1 \beta$ and IL- $1 \beta+$ COS groups after $24 \mathrm{~h}(P>0.05)$. The cell viability in the IL- $1 \beta+E V s$ and IL- $1 \beta+E V s-C O S$ groups was increased significantly compared to that in the IL-1 $\beta$ group $(P<0.05$, Fig. $2 \mathrm{~A})$. After being cultured for 48 and $72 \mathrm{~h}$, cell viability in the IL- $1 \beta+\mathrm{COS}$, IL- $1 \beta+E V s$, and IL- $1 \beta+E V s-C O S$ groups was remarkably enhanced compared to that in the IL-1 $\beta$ group $(P<0.05)$. Meanwhile, cell viability in the IL- $1 \beta+E V s-C O S$ group was enhanced more significantly than that in the IL- $1 \beta+C O S$ group $(P<0.05$, Fig. $2 \mathrm{~A})$. After culturing for $48 \mathrm{~h}$ or $72 \mathrm{~h}$, the cell viability in the IL- $1 \beta+$ EVs-COS group was also significantly higher than that in the IL- $1 \beta+$ EVs group $(P<0.05$, Fig. 2A). Based on these results, chondrocytes treated for $48 \mathrm{~h}$ were selected for subsequent experiments.
Flow cytometry was used to determine cell apoptosis in chondrocytes treated with different treatments. The cell apoptotic rates in the control and IL- $1 \beta$ groups were $2.99 \pm 0.08 \%$ and $21.26 \pm 1.11 \%$, respectively, which showed a significant increase in the IL- $1 \beta$ group compared with the control group $(P<0.05$, Fig. 2B). However, when IL-1 $\beta$-induced chondrocytes were treated with COS, EVs, and EVs-COS, the apoptotic rates were reduced to $9.12 \pm 0.49 \%, 7.64 \pm 0.37 \%$, and $6.94 \pm 0.34 \%$, respectively (Fig. 2B). Furthermore, cell apoptosis in the IL- $1 \beta+E V s-C O S$ group was significantly lower than that in the IL- $1 \beta+\operatorname{COS}$ group $(P<0.05$, Fig. $2 \mathrm{~B})$. These results indicate that IL-1 $\beta$ could significantly suppress the viability of chondrocytes and promote cell apoptosis, while EVs-COS reversed the changes in cell viability and apoptosis induced by IL-1 $\beta$. In addition, the cell viability and apoptosis changes of EVs-COS were higher than those of COS.

\section{Effects of EVs-COS on cell migration of chondrocytes}

The role of EVs-COS in the migration of IL- $1 \beta$-induced chondrocytes was further investigated using Transwell and scratch tests. Transwell results showed that IL- $1 \beta$ significantly decreased the cell number compared with the control group $(P<0.05)$, whereas COS, EVs, and EVs-COS evidently increased the reduction in cell number induced by IL-1 $\beta(P<0.05)$. The cell number in the IL- $1 \beta+E V s-C O S$ group was significantly higher than that in the IL- $1 \beta+\operatorname{COS}$ group $(P<0.05$, Fig. $3 \mathrm{~A})$. The results of the scratch test were similar to those of the Transwell test (Fig. 3B). Both Transwell and scratch tests indicated that IL-1 $\beta$ could inhibit chondrocyte migration, while EVs-COS promoted the low migration of chondrocytes caused by IL-1 $\beta$.

\section{RT-qPCR and western blot analyses}

To explore the underlying molecular mechanisms, the expression levels of IL-1 $\beta$, COL1A1, COL2A1, OPN, ALP, OCN, RUNX2, c-Myc, p53, Bcl2, Akt1, and PI3K were determined using RT-qPCR and Western blotting. Compared with the control group, the mRNA expression of $I L-1 \beta$ was significantly upregulated after IL-1 $\beta$ induction $(P<0.05)$, whereas it was significantly downregulated after COS, EVs, and EVs-COS treatment compared with the IL- $1 \beta$ group $(P<0.05)$. Furthermore, the actions of EVs-COS were higher than that of COS $(P<0.05$, Fig. 4A). The mRNA expression of COL1A1 and COL2A1 was evidently downregulated in the IL- $1 \beta$ group compared with the control group $(P<0.05)$, while they were observably upregulated in the IL- $1 \beta+C O S$, IL- $1 \beta+E V s$, IL- $1 \beta+E V s-C O S$ groups compared with 


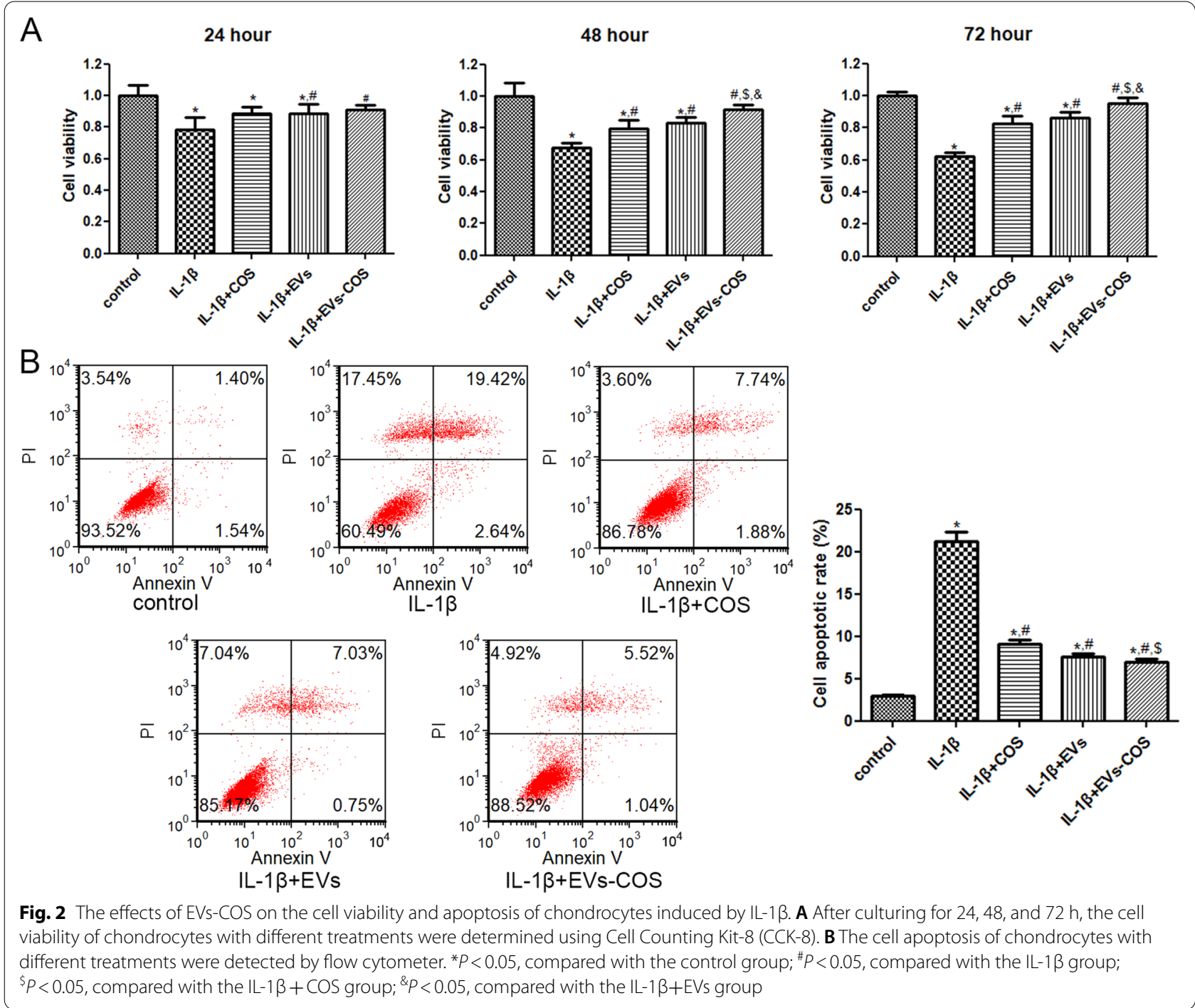

the IL-1 $\beta$ group $(P<0.05)$. In addition, the mRNA levels in the IL- $1 \beta+E V s$ and IL- $1 \beta+E V s-C O S$ groups were both significantly higher than those in the COS group $(P<0.05$, Fig. $4 \mathrm{~B}, \mathrm{C})$. The trend of OPN mRNA expression was similar to that of $I L-1 \beta$ mRNA expression (Fig. 4D). Interestingly, the mRNA expression of $A L P$ was significantly downregulated in the IL-1 $\beta$ group compared to that in the control group $(P<0.05)$ and continued to decline after COS, EVs, and EVs-COS treatments $(P<0.05$, Fig. 4E). The mRNA expression of $O C N$ and $R U N X 2$ was markedly decreased in the IL- $1 \beta$ group compared to that in the control group $(P<0.05)$, while COS, EVs, and EVs-COS properly restored their expression (Fig. 4F, G). The mRNA expression of Akt1 and PI3K was similar to that of OCN mRNA expression (Fig. 4H, I). Furthermore, compared with the control group, the mRNA expression of $c-M y c$ was significantly downregulated after IL-1 $\beta$ induction $(P<0.05)$, while it was notably increased in the COS, EVs, and EVsCOS groups $(P<0.05$, Fig. $4 \mathrm{~J})$. Nevertheless, the trend of $p 53$ mRNA expression was opposite to that of $c-M y c$ expression (Fig. 4K). For Bcl2, the tendency of its mRNA expression was in line with that of $c-M y c$ mRNA expression (Fig. 4L). Additionally, the results of the Western blot analyses of COL1A1, COL2A1, ALP, OPN, OCN, RUNX2, c-Myc, p53, and PI3K protein expression levels were similar to those of the RT-qPCR (Fig. $4 \mathrm{M}, \mathrm{N}$ ). The level of p-Akt/Akt in the IL-1 $\beta$ group was significantly downregulated compared to that in the control group $(P<0.05)$, while after treatment with $\mathrm{COS}$, EVs, and EVs-COS, its level was significantly upregulated $(P<0.05)$, and the effect of EVs-COS was better than that of $\operatorname{COS}(P<0.05$, Fig. 4M, N). 


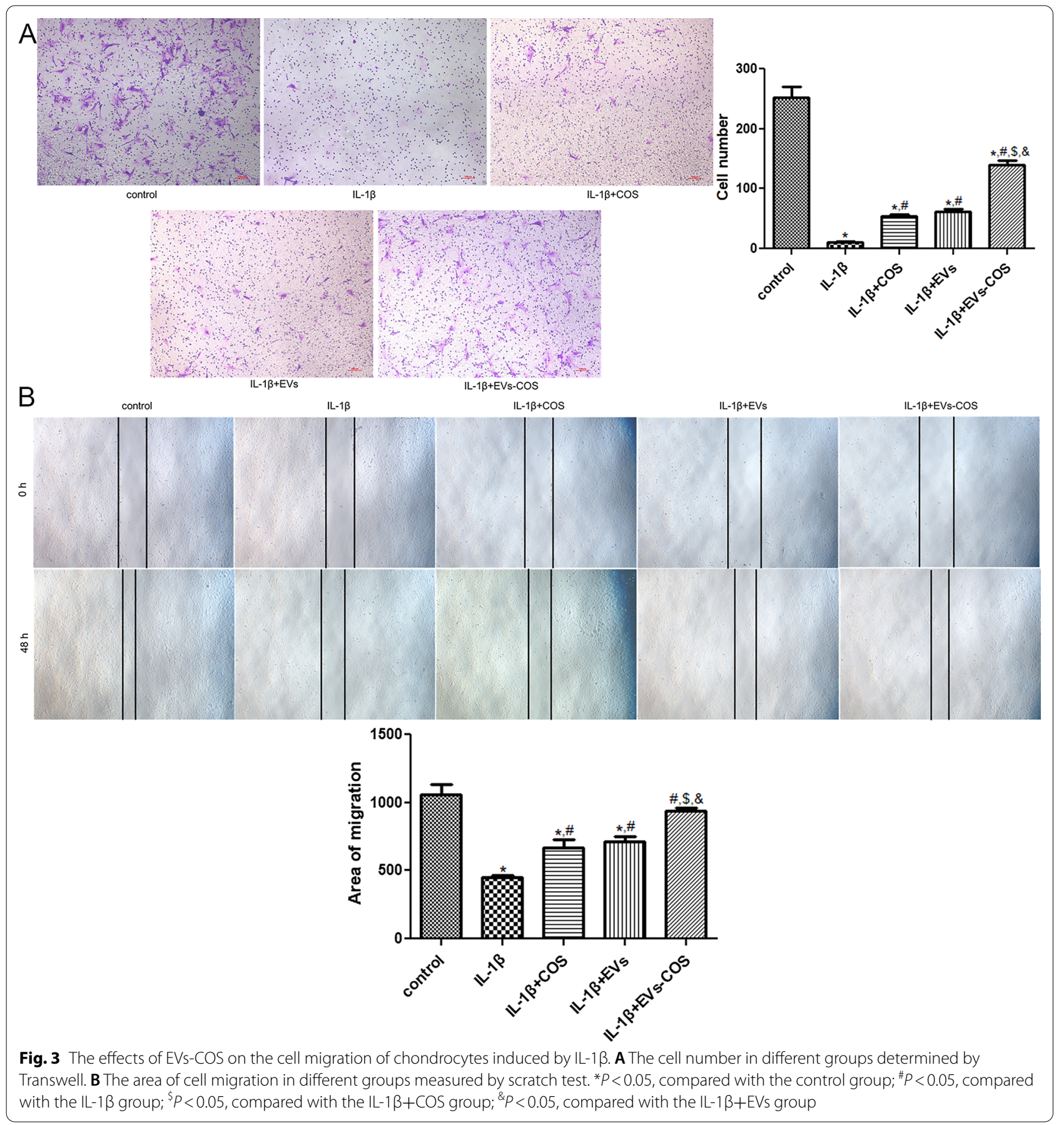

(See figure on next page.)

Fig. 4 The effects of EVs-COS on the expression levels of related genes and proteins in the chondrocytes with different treatments. The mRNA

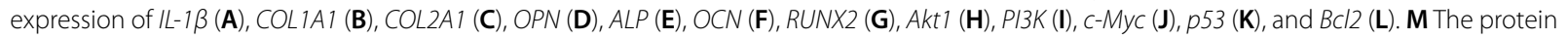
bands determined by Western blot. (N) The protein expressions of COL1A1, COL2A1, ALP, OCN, OPN, RUNX2, p-Akt/Akt, PI3K, c-Myc, and p53. ${ }^{*} P<0.05$, compared with the control group; ${ }^{\#} P<0.05$, compared with the IL-1 $\beta$ group; ${ }^{p} P<0.05$, compared with the IL-1 $\beta+\operatorname{COS}$ group; \& $P<0.05$, compared with the IL-1 $\beta+E V$ s group 

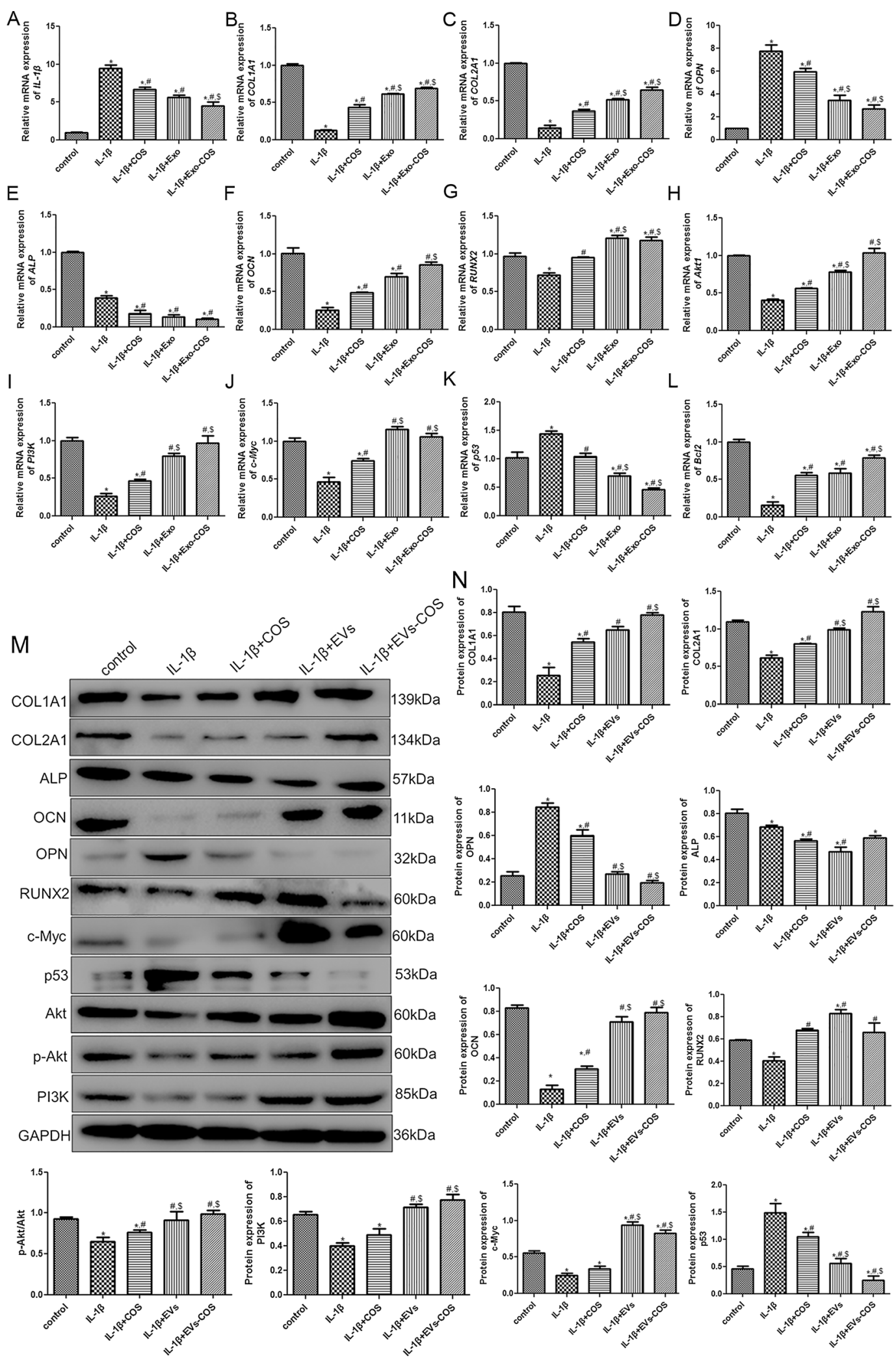

Fig. 4 (See legend on previous page.) 


\section{HE staining analysis and immunohistochemical staining of collagen I and collagen II}

Cartilage tissues isolated from the rats in the control, injury model, COS, EVs, and EVs-COS groups, and histological changes associated with cartilage injury and EVsCOS treatment in rats were investigated by HE staining of cartilage tissues. The control group showed normal cartilage tissue without infiltration of inflammatory cytokines (Fig. 5A). After modeling, the cartilage tissues in the model group were damaged and swollen, the cartilage matrix was degenerated, and inflammatory cytokines were infiltrated. Cartilage tissue injury was alleviated in the COS, EVs, and EVs-COS groups, and EVs-COS had a better treatment effect on cartilage tissue injury (Fig. 5A).

Subsequently, the expression of collagen I and II was determined using immunohistochemical staining. As shown in Fig. 5B, the expression of COL1A1 in the model and COS groups was significantly lower than that in the control $(P<0.05)$. EVs and EVs-COS significantly increased the expression of COL1A1 compared to the model group $(P<0.05)$. Meanwhile, COL2A1 expression was dramatically downregulated in the model group compared to that in the control group $(P<0.05)$, while COS, EVs, and EVs-COS markedly upregulated its expression $(P<0.05)$. No significant difference was observed among the control, EV, and EVs-COS groups $(P>0.05$, Fig. 5C). These results indicated that the expression of COL1A1 and COL2A1 could be downregulated after cartilage injury, and that EVs-COS could reverse the trends (i.e., expression reduction) caused by cartilage injury.

\section{Screening of DEGs in the same expression pattern and functional analyses}

After comparison, a total of 2,091, 503, and 412 DEGs were identified between the COS and model groups, between EVs-COS and model groups, as well as between EVs-COS and COS groups, respectively (Additional file 3: Figure S3). Thereafter, by comparing the DEGs between COS vs. model, EVs-COS vs. model, and EVs-COS vs. $\mathrm{COS}$, and retaining DEGs in at least two comparison groups, 760 DEGs were obtained for further expression trend clustering analysis (Additional file 4: Table S1).

Afterwards, the Mfuzz algorithm was used to analyze the expression patterns of the obtained 760 DEGs, and the expression trend of the target genes under different treatments was observed. Four expression patterns, clusters 1, 2, 3, and 4, were clustered (Fig. 6), and the DEGs in each are shown in Additional file 4: Table S1.

In addition, the DEGs in each cluster were used for the BP and KEGG enrichment analyses (Additional file 5: Table S2). It was found that the DEGs in cluster 1 were significantly enriched in allograft rejection, signaling pathways regulating pluripotency of stem cells, phagosomes, negative regulation of canonical Wnt signaling pathway (SFRP5, SOX9, and GLI1), cellular response to transforming growth factor beta stimulus, regulation of cell proliferation, and cartilage condensation (Fig. 7A). The DEGs in cluster 2 were strongly associated with ECM-receptor interaction, focal adhesion, PI3K-Akt signaling pathway (COL1A1, COL3A1, ITGA4, BAD, and $F O X O 3$ ), and proteolysis (Fig. 7B). Meanwhile, the DEGs in cluster 3 were involved in adrenergic signaling in cardiomyocytes, AMPK signaling pathway $(P P P 2 R 3 A$, $C P T 1 B$, and $A C A C B$ ), and positive regulation of potassium ion transport (Fig. 7C). Lastly, the DEGs in cluster 4 played important roles in the regulation of actin cytoskeleton, MAPK signaling pathway (CACNA1S, CACNG1, and $M A P K 12)$, pentose phosphate pathway, sarcomere organization, muscle contraction, skeletal muscle tissue development, and myofibril assembly (Fig. 7D).

\section{Validation of sequencing results by RT-qPCR}

Five DEGs, including three upregulated and two downregulated in the COS and EVs-COS groups, were chosen for the validation of sequencing results by using RTqPCR. It is clear that the expression levels of $V p s 13 a$ and Itga1 were significantly upregulated in the COS and EVsCOS groups compared with the model group $(P<0.05$, Fig. 8A, B). Furthermore, the expression of $I f 27 l 2 b$ and Gli1 in the COS and EVs-COS groups were obviously lower than those in the model group $(P<0.05$, Fig. 8D, E). These results indicated that the tendency of $V p s 13 a$, Itga1, Ifi27l2b, and Gli1 expression determined by RTqPCR were consistent with those analyzed by sequencing. However, there was no significant difference in Birc6 expression among the model, COS, and EVs-COS groups $(P>0.05$, Fig. $8 C)$. All these results implied that the consistency rate of sequencing findings and RT-qPCR results was $80 \%$, which represents a relatively high reliability.

\section{Discussion}

OA is the injury of articular cartilage caused by multiple factors, such as mechanical trauma or inflammation, which seriously affects people's health and quality of life [5]. EVs are carriers of many bioactive substances and play important roles in cell-to-cell communication [29]. Chitin and its derivative chitosan have been reported as bone repair materials that promote osteoblast growth and healing [22]. In this study, COS was packaged into rat AMSC-derived EVs, and the effects of EVs-COS on cartilage injury repair both in vitro and in vivo were explored. Previous studies have suggested that MSC-derived EVs can attenuate OA [30], and that COS can improve cartilage damage, thus, preventing and treating OA [31]. Our research found that EVs-COS could enhance the viability 


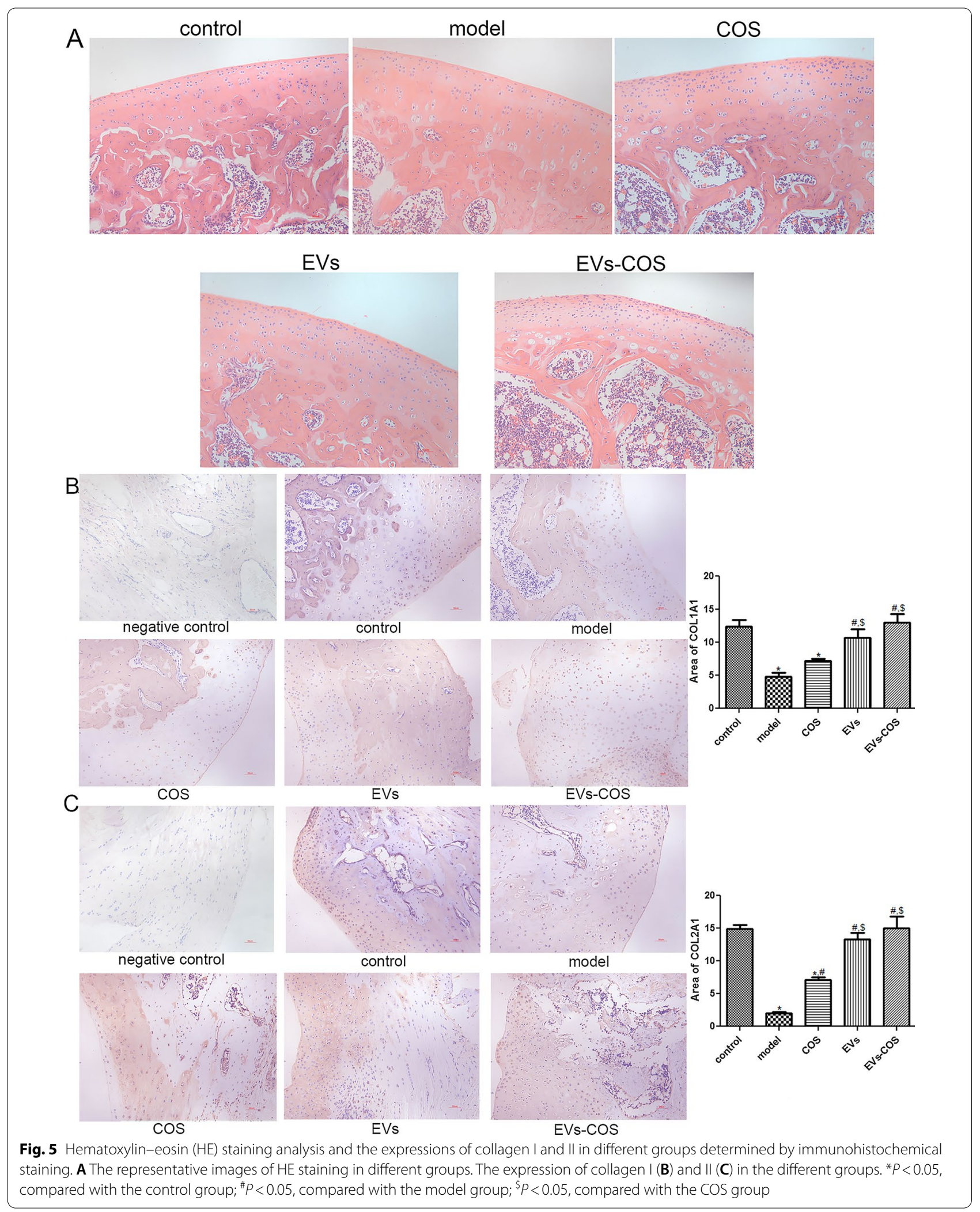



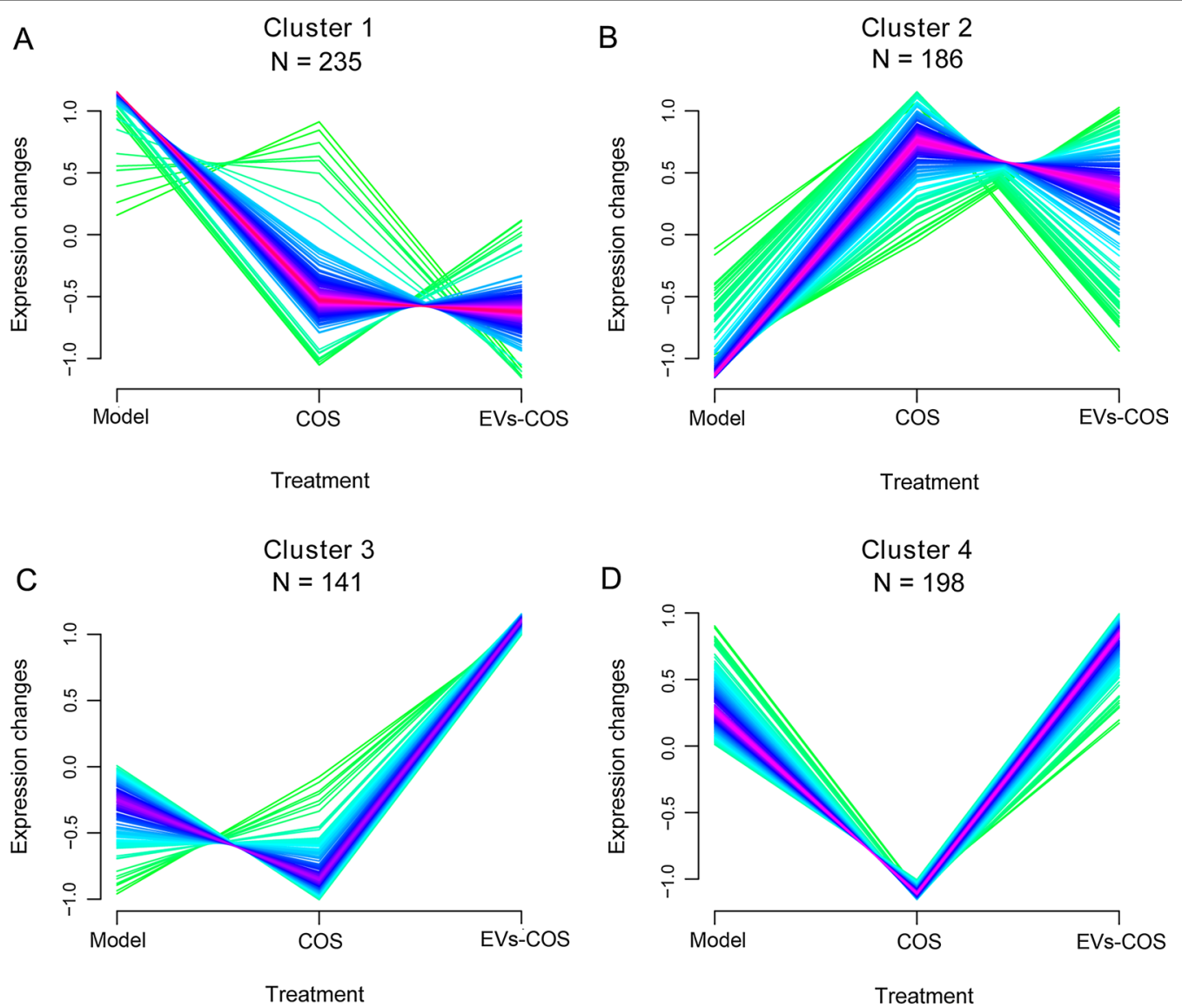

Fig. 6 Clustering diagram of expression patterns of the obtained differentially expressed genes (DEGs) based on Mfuzz algorithm

and migration of chondrocytes induced by IL- $1 \beta$ and suppress their apoptosis. A previous study demonstrated that human MSC-derived EVs can relieve IL- $1 \beta$-induced chondrocyte viability repression and apoptosis induction, thus alleviating chondrocyte damage and OA [32]. Another study has shown that hyaluronic acid/chitosan nanoparticles can deliver curcuminoids to increase cell proliferation while suppressing apoptosis and inflammatory response in the chondrocytes of knee OA [33]. Taken together, we speculated that EVs-COS may promote cartilage injury repair and ameliorate OA by promoting the viability and migration of chondrocytes and inhibiting cell apoptosis.

To investigate the mechanisms of the treatment of OA by EVs-COS in vitro, RT-qPCR and Western blotting were performed to measure the expression levels of related genes and proteins. After cartilage damage, IL- $1 \beta$, OPN, and p53 were significantly upregulated, while COL1A1, COL2A1, OCN, RUNX2, p-Akt/Akt, PI3K, $\mathrm{c}-\mathrm{Myc}$, and $\mathrm{Bcl} 2$ were markedly downregulated. Meanwhile, COS and EVs-COS could reverse the expression induced by cartilage injury, and the actions of EVs-COS were stronger. IL-1 $\beta$, a pro-inflammatory cytokine, has been used to establish an inflammatory model of chondrocytes in vitro [34]. COL1A1 and COL2A1, encoding type I and II collagen, are chondrocyte-specific markers that are essential for linear growth, bone development, and the structural framework of cartilage [35]. A study by Jain et al. reported that IL- $1 \beta$ pre-treatment inhibited COL1A1 and COL2A1 expression in chondrocytes, while lyophilized platelet-rich plasma treatment increased the level of COL1A1 [36]. OCN, OPN, and RUNX2 are osteoblast-related genes, and their expression is closely related to the maturation and differentiation of osteoblasts [37]. RUNX2 has been reported to be a crucial gene in osteogenesis that induces the expression of OPN and OCN [37]. A previous study showed that lipopolysaccharide (LPS) significantly reduced osteoblastic differentiation in MC3T3-E1 cells by downregulating RUNX2, OCN, and OPN expression [38], while resveratrol promoted LPS-induced MC3T3-E1 cell differentiation and increased the expression of RUNX2, OCN, and OPN. P53, a well-known apoptotic gene, is involved in the cell cycle, DNA repair, cell apoptosis, and senescence 


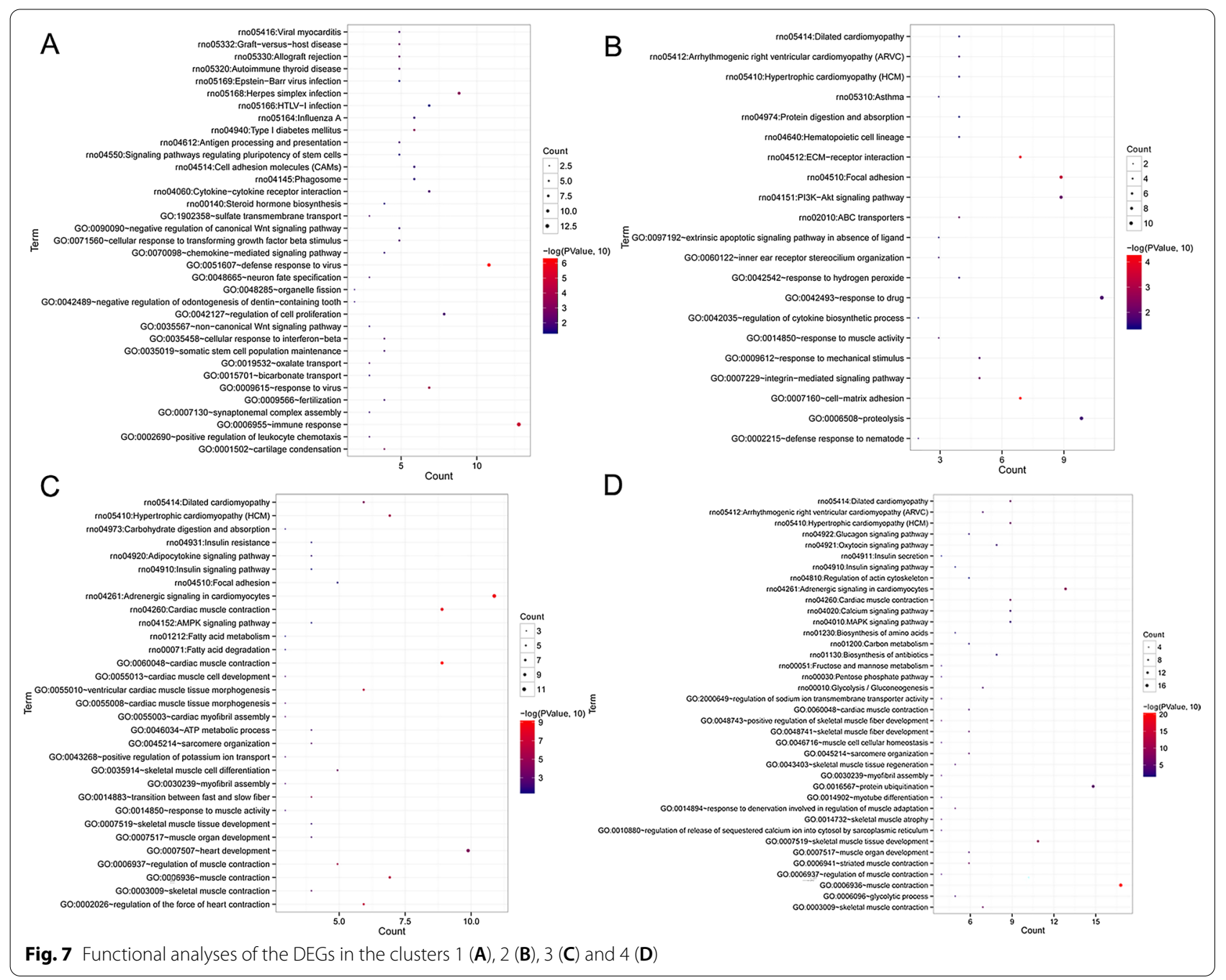

[39]. c-Myc is a primary regulator of cell proliferation and transformation, and its abnormal expression is the basis of many cancers [40]. Bcl2, an anti-apoptotic factor, has been reported to participate in apoptosis and the growth and differentiation of chondrocytes [41]. Mei et al. indicated that PRMT5 knockdown could inhibit the growth and migration of endometrioid adenocarcinoma cells and promote cell apoptosis by downregulating c-Myc and $\mathrm{Bcl} 2$, and upregulating p53 [42]. Akt is the main effector of PI3K signal transduction and modulates a variety of pathways, such as the inhibition of cell apoptosis, stimulation of cell growth, and regulation of cell metabolism [43]. Bakuchiol promotes chondrocyte proliferation and injured cartilage remodeling through the PI3K/Akt and ERK1/2 pathways [44]. These findings are consistent with our experimental results, and suggest that EVsCOS could affect viability, apoptosis, and migration of chondrocytes by regulating chondrocyte-specific genes (COL1A1 and COL2A1), osteoblast-related genes (OCN,
OPN, and RUNX2), apoptosis-related genes (c-Myc, p53, and $\mathrm{Bcl} 2)$, and the Akt/PI3K pathway, thereby improving cartilage injury repair and OA.

Subsequently, an in vivo cartilage injury model was constructed, and chondrocytes were isolated from different groups. The expression of COL1A1 and COL2A1 was determined using immunohistochemical staining, and the results of COL1A1 and COL2A1 in vivo were consistent with those in vitro. Afterwards, the chondrocytes in the model, COS, and EVs-COS groups were used for sequencing, and a total of 760 DEGs were identified, which were clustered into four expression patterns (clusters $1,2,3$, and 4 ). The DEGs in all clusters were associated with negative regulation of the canonical Wnt, PI3K-Akt, AMPK, and MAPK signaling pathways. The canonical Wnt signaling pathway, mediated by $\beta$-catenin, has been reported to promote the differentiation of osteoblasts, bone formation, and bone resorption [45]. An experimental OA mouse model was established, which 

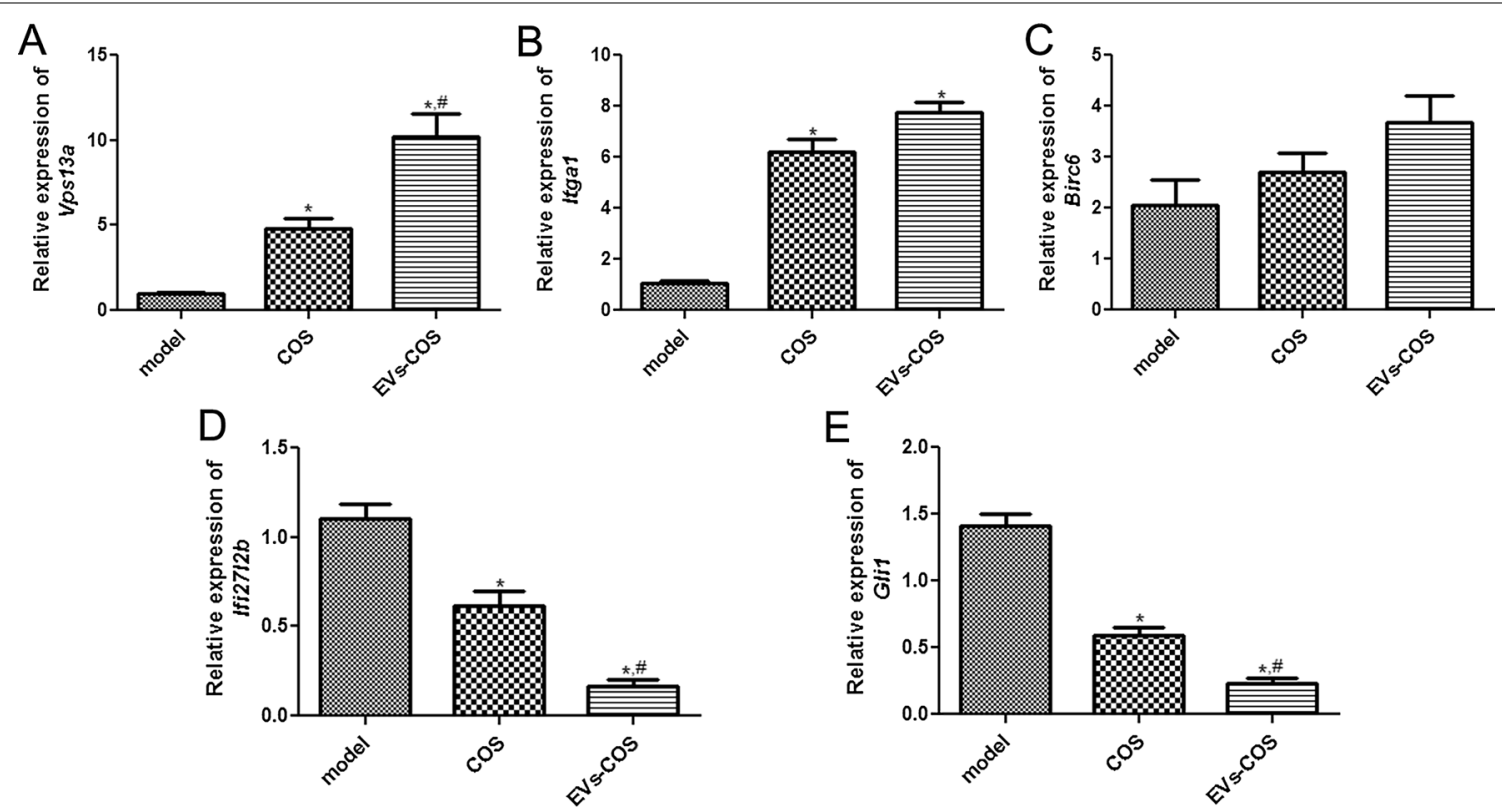

Fig. 8 Validation of DEGs by real-time quantification PCR. The mRNA expression levels of Vps13a (A), Itgal (B), Birc6 (C), Ifi27l2b (D), and Gli1 (E). ${ }^{*} P<0.05$, compared with the model group; ${ }^{\#} P<0.05$, compared with the COS group

revealed that the inhibition of the $\mathrm{Wnt} / \beta$-catenin signaling pathway improved the severity of OA and reduced the degeneration of cartilage and synovitis in vivo [46]. Our in vitro experiments have shown that EVs-COS can affect cartilage injury repair through the PI3K-Akt signaling pathway, a finding confirmed by the in vivo results. It has been reported that the AMPK signaling pathway plays important roles in regulating cellular energy balance, resisting oxidative stress, and alleviating inflammation [47]. A previous study reported that LPS induced the activation of the AMPK/NF- $\mathrm{kB}$ pathway and higher levels of chondrocyte apoptosis, while miR-137 overexpression inhibited the AMPK/NF- $\mathrm{kB}$ pathway and reversed OA development [48]. Additionally, the activation of the MAPK signaling pathway can contribute to the expression of pro-inflammatory cytokines and chemokines in chondrocytes of OA, while blockage of this pathway can inhibit chondrocyte apoptosis and prevent the recruitment of inflammatory cells that may lead to the degradation of cartilage [49]. These findings confirm that EVs-COS can promote cartilage injury repair and relieve OA through negative regulation of the canonical Wnt, PI3K-Akt, AMPK, and MAPK signaling pathways.

Through sequencing, we also found that SFRP5, SOX9, and GLI1 were significantly enriched in the negative regulation of the canonical Wnt signaling pathway; COL1A1, COL3A1, ITGA4, BAD, and FOXO3 were significantly enriched in the PI3K-Akt signaling pathway; PPP2R3A, $C P T 1 B$, and $A C A C B$ were significantly enriched in the
AMPK signaling pathway; and CACNA1S, CACNG1, and $M A P K 12$ were significantly enriched in the MAPK signaling pathway. A previous study has shown that the level of SFRP5 is closely related to bone density and bone formation [50]. SOX9, a key transcription factor in chondrocytes, has been widely reported and studied for cartilage development [51]. GLI1, COL1A1, COL3A1, and FOXO3 have also been reported to be related to cartilage injury repair and the occurrence and progression of OA [5254]. Therefore, the hypothesis that EV-COS may promote cartilage injury repair by regulating SFRP5, SOX9, GLI1, COL1A1, COL3A1, and FOXO3 may be correct.

However, few reports have shown the roles of ITGA4, $B A D, P P P 2 R 3 A, C P T 1 B, A C A C B, C A C N A 1 S, C A C N G 1$, and $M A P K 12$ in cartilage injury repair. ITGA4 encodes a member of the integrin $\alpha$ chain family, and its high level is associated with many diseases, such as chronic lymphocytic leukemia [55], gastrointestinal stromal tumors [56] and colorectal cancer [57]. BAD plays a key role in mitochondria-dependent apoptosis, and its inactivation contributes to the development of rheumatoid arthritis by conferring apoptosis resistance in synovial sub lining macrophages [58]. PPP2R3A, a major serine/threonine phosphatase, is involved in a variety of cellular processes, including cell apoptosis, proliferation, DNA repair, and autophagy [59], and its knockdown inhibits the proliferation, migration, and invasion of hepatoma carcinoma cells [60]. $C P T 1 B$ and $A C A C B$ are genes related to fatty acid oxidation [61] and are associated with obesity and 
diabetes. CACNA1S and CACNG1, calcium channel related genes, could serve as novel therapeutic targets for remodeling the extracellular matrix of aging lamina propria [62]. MAPK12, which may be a risk factor for the prognosis of bladder cancer patients [63], encodes p38 $\gamma$, which is mainly expressed in the skeletal muscle. Combining these studies with our results, it can be inferred that ITGA4, BAD, PPP2R3A, CPT1B, ACACB, CACNA1S, CACNG1, and MAPK12 may play important roles in promoting cartilage injury repair by EVs-COS. However, the specific effects of these genes on OA should be further verified both in vitro and in vivo.

\section{Conclusions}

EVs-COS could facilitate cartilage injury repair and have better protective effects on OA by promoting the viability and migration of chondrocytes, suppressing cell apoptosis, and regulating COL1A1, COL2A1, OCN, OPN, RUNX2, c-Myc, p53, Bcl2, and the Akt/PI3K pathway. Sequencing revealed that negative regulation of the canonical Wnt, PI3K-Akt, AMPK, and MAPK signaling pathways may be strongly associated with cartilage injury repair. SFRP5, SOX9, GLI1, COL1A1, COL3A1, FOXO3, GA4, BAD, PPP2R3A, CPT1B, ACACB, CACNA1S, $C A C N G 1$, and MAPK12 may be potential targets for EVCOS-promoting cartilage injury repair. Our findings have revealed the underlying pathogenesis of $\mathrm{OA}$ and laid the foundation for the therapy of cartilage injuries and damage-based OA with EVs-COS as a novel potential drug.

\section{Methods}

\section{Isolation and identification of EVs}

Rat AMSCs were purchased from Cyagen Biosciences Inc. (Guangzhou, China). Rat AMSCs were cultured in $\alpha$-minimum essential medium ( $\alpha$-MEM; Thermo Fisher Scientific, Waltham, MA, USA), supplemented with $10 \%$ fetal bovine serum (FBS, Thermo Fisher Scientific) and $1 \%$ penicillin/streptomycin (Thermo Fisher Scientific), and maintained in an incubator with $5 \%$ carbon dioxide at $37^{\circ} \mathrm{C}$.

The extraction methods for EVs from rat AMSCs have been described previously [64]. Briefly, rat AMSCs were cultured in medium with serum but without EVs for $48 \mathrm{~h}$, and the cell supernatants of rat AMSCs were collected. The obtained cell supernatants were transferred to a new $50 \mathrm{~mL}$ tube, and centrifuged at $500 \times g$ for $4 \mathrm{~min}$ at $4{ }^{\circ} \mathrm{C}$. The supernatant was then transferred to a new tube and centrifuged at $2000 \times g$ for $30 \mathrm{~min}$ at $4{ }^{\circ} \mathrm{C}$. The supernatant was then mixed with the same amount of pre-cooled polyethylene glycol (PEG, 16\%) and incubated at $4{ }^{\circ} \mathrm{C}$ overnight. After centrifugation at $10,000 \times g$ for $60 \mathrm{~min}$ at $4{ }^{\circ} \mathrm{C}$, the supernatant was discarded, and $1 \mathrm{~mL}$ phosphate buffer saline (PBS) was added to the sediment. After blending, the mixture was centrifuged at $100,000 \times g$ for $70 \mathrm{~min}$ at $4{ }^{\circ} \mathrm{C}$. The sediment was resuspended in $200 \mu \mathrm{L}$ PBS, and the EVs were stored at $-80{ }^{\circ} \mathrm{C}$ for subsequent experiments.

The concentrations of EVs were measured using a BCA assay kit (Thermo Fisher Scientific), according to the manufacturer's instructions. Subsequently, a Nanosight NS300 particle size analyzer (NTA; Malvern Panalytical, Malvern, UK) was used to determine the particle distribution of EVs following the method described by Soares Martins et al. [29]. The morphology of isolated EVs was visualized by transmission electron microscopy (TEM, JEOL LTD, Peabody, MA, USA) according to a previous study [65]. Furthermore, the protein expression of CD63, TSG101, CD9, and calnexin were detected in the EVs and cells via Western blotting with their corresponding antibodies (1:2000) [66].

\section{Binding of EVs with COS}

The concentration of EVs was adjusted to $40 \mu \mathrm{g} / \mathrm{mL}$ using Dulbecco's modified Eagle's medium (DMEM, Thermo Fisher Scientific), and $640 \mu \mathrm{g} / \mathrm{mL}$ COS (Tokyo Chemical Industry, TCI, Tokyo, Japan) was prepared with DMEM. Thereafter, EVs were mixed with COS in equal volumes, and the mixture was incubated with shaking at $37{ }^{\circ} \mathrm{C}$ for $1 \mathrm{~h}$. After centrifugation at $120,000 \times g$ for $60 \mathrm{~min}$, the sediment was resuspended in $200 \mu \mathrm{L}$ PBS, and EVsCOS conjugates were obtained. A TEM was then used to determine the morphology of the EVs-COS, and NTA was used to measure the size distribution of the EVs-COS.

\section{Isolation and culture of rat chondrocytes}

Rat chondrocytes were isolated from the cartilage tissues of SPF Wistar rats weighing $180 \pm 20 \mathrm{~g}$, as described previously [34]. Briefly, the rats were killed by cervical dislocation, and cartilage tissues from the knee were collected under sterile conditions. After washing thrice with PBS, the cartilage tissues were cut into small pieces $\left(1 \mathrm{~mm}^{3}\right)$, and then $0.2 \%$ type II collagenase solution (Beyotime Biotechnology, Shanghai, China): $0.25 \%$ trypsin solution (Beyotime Biotechnology; v/v, 4:1; including DNase) was added. After digestion at $37^{\circ} \mathrm{C}$ for $6 \mathrm{~h}$, DMEM/F12 medium (Thermo Fisher Scientific) was added to terminate the digestion. After filtering, the collected chondrocyte suspension was centrifuged at $1000 \mathrm{rpm}$ for $5 \mathrm{~min}$, and the chondrocytes were cultured in DMEM/ F12 medium supplemented with 10\% FBS and 1\% penicillin/streptomycin. Afterwards, the chondrocytes were 
inoculated into a $25 \mathrm{~cm}^{2}$-cell culture plate and passaged when the cell confluence reached $80-90 \%$.

\section{Immunohistochemistry staining}

The chondrocytes were characterized by immunohistochemical staining for type II collagen. The chondrocytes were washed with PBS and fixed with $4 \%$ paraformaldehyde for $20 \mathrm{~min}$. After washing thrice with PBS, 0.5\% Triton X-100 (Sigma-Aldrich, USA) was added, and the mixture was incubated at $22 \pm 3{ }^{\circ} \mathrm{C}$ for $15 \mathrm{~min}$. After washing, $3 \% \mathrm{H}_{2} \mathrm{O}_{2}$ was added, and the resulting mixture was incubated at $22 \pm 3{ }^{\circ} \mathrm{C}$ for $15 \mathrm{~min}$. The cells were then washed and then incubated with anti-collagen II antibody (1:1000, Proteintech, Chicago, USA) or anti-collagen I antibody (1:1000, NOVUS) overnight. Afterwards, the cells were washed and then treated with horseradish peroxidase (HRP)-labeled secondary antibody (Goat anti-rabbit IgG, Jackson ImmunoResearch Laboratories, Inc.) for $1 \mathrm{~h}$. After washing, the cells were incubated with diaminobenzidine (DAB, Beyotime Biotechnology) for $5 \mathrm{~min}$, and the cells were then redyed with hematoxylin (Sigma-Aldrich). The color of the cells was observed under a microscope at $200 \times$ magnification.

\section{Co-culture of EVs or EVs-COS and chondrocytes}

EVs and EVs-COS were labeled with PKH67 (green fluorescence) using a PKH67 staining kit (MINI67-1KT, Sigma-Aldrich) following the manufacturer's protocols. Briefly, Diluent C $(900 \mu \mathrm{L})$ was added to the EVs $(100$ $\mu \mathrm{L})$ or EVs-COS $(100 \mu \mathrm{L})$, and then PKH67 $(4 \mu \mathrm{L})$ was added. After incubation at $22 \pm 3{ }^{\circ} \mathrm{C}$ for $5 \mathrm{~min}, 2 \mathrm{~mL}$ of $1 \%$ BSA was added to bind excess dye. After centrifugation at $120,000 \times g$ for $60 \mathrm{~min}$ at $4{ }^{\circ} \mathrm{C}$, the sediment was resuspended in $300 \mu \mathrm{L}$ PBS, and PKH67-labeled EVs and EVs-COS were successfully prepared.

Afterwards, the chondrocytes were seeded in a 24-well plate at a density of $3 \times 10^{4}$ cells/well and cultured in serum-free medium overnight. The next day, $20 \mu \mathrm{L}$ PKH67-labeled EVs and EVs-COS were added to the cells. After co-culturing for $48 \mathrm{~h}$, the cells were washed with PBS and fixed with $4 \%$ paraformaldehyde for $20 \mathrm{~min}$. After washing, the cells were treated with $0.1 \%$ Triton X-100 for $5 \mathrm{~min}$. Afterwards, the cells were washed, added with $1 \% \mathrm{BSA}$, and incubated at $22 \pm 3{ }^{\circ} \mathrm{C}$ for $30 \mathrm{~min}$. After removing the $1 \% \mathrm{BSA}$, the cells were stained with actin red (red fluorescence) in the dark at $22 \pm 3{ }^{\circ} \mathrm{C}$ for $20 \mathrm{~min}$. Thereafter, the cells were stained with DAPI (Thermo Fisher Scientific), and images were taken using a laser scanning confocal microscope (TCS SP8, Leica Microsystems, Inc., USA).

\section{Cell viability and cell apoptosis assays}

The chondrocytes were seeded into 96-well plates and divided into five groups: control, IL- $1 \beta$, IL- $1 \beta+C O S$, IL- $1 \beta+E V s$, and IL- $1 \beta+E V s-C O S$ groups. The cells in the IL- $1 \beta$, IL- $1 \beta+C O S$, IL- $1 \beta+E V s$, and IL- $1 \beta+E V s-C O S$ groups were treated with $10 \mathrm{ng} / \mathrm{mL}$ IL- $1 \beta$ for $24 \mathrm{~h}$ first, and then with PBS, $320 \mu \mathrm{g} / \mathrm{mL}$ COS, $20 \mu \mathrm{g} / \mathrm{mL}$ EVs, and EVs-COS $(20 \mu \mathrm{g} / \mathrm{mL} \mathrm{EVs}+320 \mu \mathrm{g} / \mathrm{mL}$ COS $)$, each, for another $24 \mathrm{~h}, 48 \mathrm{~h}$, and $72 \mathrm{~h}$. Cells in the control group were not treated.

The cell viability of the chondrocytes was examined using a Cell Counting Kit-8 (CCK-8, Beyotime Biotechnology) according to the manufacturer's recommendations. Briefly, $10 \mathrm{~mL}$ of CCK- 8 reagent was added to each well and incubated for $2 \mathrm{~h}$. The absorbance at $450 \mathrm{~nm}$ was determined using a microplate reader (Multiskan MK3; Thermo Fisher Scientific).

After culturing for $48 \mathrm{~h}$, the cell suspension was harvested and used for the cell apoptosis assay using the Annexin V-FITC/PI apoptosis assay kit (Beyotime Biotechnology). The harvested cell suspension was centrifuged at $1000 \mathrm{rpm}$ for $5 \mathrm{~min}$ and resuspended in $1 \times$ binding buffer $(100 \mu \mathrm{L})$. Afterwards, $5 \mu \mathrm{L}$ each of Annexin V-FITC and propidium iodide (PI) were added to the cells. After incubation at $22 \pm 3{ }^{\circ} \mathrm{C}$ in the dark for $15 \mathrm{~min}, 400 \mu \mathrm{L}$ of $1 \times$ binding buffer was added to the mixture. Images of apoptosis were acquired using a flow cytometer.

\section{Cell migration assay}

Transwell and scratch tests were performed to evaluate the cell migration. For Transwell, chondrocytes with different treatments were collected and resuspended in $0.1 \%$ BSA medium. Afterwards, $200 \mu \mathrm{L}$ of cell suspension was added to the upper chamber of the Transwell chambers (pore size $8 \mu \mathrm{m}$; Guangzhou Jet Bio-Filtration Co., Ltd., Guangzhou, China), while complete medium was added to the lower chamber. After culturing for another $24 \mathrm{~h}$, the chambers were removed, and the supernatant was removed. The cells were then washed and then fixed with $4 \%$ paraformaldehyde for $10 \mathrm{~min}$. After washing with PBS, the cells were stained with crystal violet (Beyotime Biotechnology) at $22 \pm 3{ }^{\circ} \mathrm{C}$ for $20 \mathrm{~min}$. After washing the excess dye and drying, the cells were observed and photographed under an inverted microscope.

For the scratch test, the chondrocytes were seeded in a 6 -well plate at a density of $5 \times 10^{5}$ cells/well, and the cells were subjected to different treatments. The cell layer was then scratched with a micropipette, and the cell medium was removed. After washing with PBS thrice, serum-free medium was added. Images of the cells were captured at $0 \mathrm{~h}$ and $48 \mathrm{~h}$. 


\section{Real-time quantitative PCR (RT-qPCR)}

Total RNA was extracted from the chondrocytes with different treatments using RNAiso Plus (TRIzol, TAKARA, Japan) according to the manufacturer's instructions. The concentrations and quality of the total RNA were measured using a microplate reader. Next, PrimeScript ${ }^{\mathrm{TM}}$ RT Master Mix (TAKARA) was used to reverse transcribe the total RNA into cDNA following the manufacturer's instructions. The RT-qPCR reaction was initiated at $95{ }^{\circ} \mathrm{C}$ for $2 \mathrm{~min}$, followed by 40 cycles of $95^{\circ} \mathrm{C}$ for $15 \mathrm{~s}$, $60{ }^{\circ} \mathrm{C}$ for $60 \mathrm{~s}, 95^{\circ} \mathrm{C}$ for $15 \mathrm{~s}, 60{ }^{\circ} \mathrm{C}$ for $60 \mathrm{~s}$, and $95{ }^{\circ} \mathrm{C}$ for 15 s. GAPDH was used as a housekeeping gene, and the sequences of all primers are shown in Table 1. Relative mRNA expression levels were calculated using the $2^{-\Delta \Delta \mathrm{Ct}}$ method [67].

\section{Western blot}

RIPA lysis buffer (Beyotime Biotechnology) was used to isolate total protein from the cells with different treatments, and the protein concentrations were determined using a BCA assay kit (Thermo Fisher Scientific). Protein samples $(20 \mu \mathrm{g})$ were separated by $10 \%$ SDS-PAGE, transferred to polyvinylidene fluoride membranes, and blocked with $5 \%$ skim milk at $37{ }^{\circ} \mathrm{C}$ for $2 \mathrm{~h}$. After washing thrice with PBST (PBS with 1\% Tween-20), the membranes were incubated with primary antibodies against c-Myc (1:1000, CST), ALP (1:500, Abcam), OCN (1:1000, NOVUS), RUNX2 (1:1000, Abcam), OPN (1:600, Abcam), Akt (1:1000, CST), p-Akt (1:2000, CST), PI3K (1:2000, Proteintech), COL1A1 (1:1000, NOVUS), COL2A1 (1:1000, Proteintech), p53 (1:1000, Proteintech), and GAPDH (1:1000, Proteintech) at $4{ }^{\circ} \mathrm{C}$ overnight. After washing, the membranes were then incubated with the secondary antibody (1: 1000, Jackson ImmunoResearch Laboratories, Inc.) at $37^{\circ} \mathrm{C}$ for $2 \mathrm{~h}$. After washing, the protein bands were visualized using ECL system.

\section{Animal experiments in vivo}

Thirty SPF female Wistar rats weighing $180 \pm 20 \mathrm{~g}$ were purchased from Shanghai SLAC Laboratory Animal Co., Ltd. (Shanghai, China). All rats were maintained at $22-25{ }^{\circ} \mathrm{C}$ and $20-25 \%$ humidity conditions, with a $12 \mathrm{~h}$ light/dark cycle. During the experiments, all rats had ad libitum access to water and food. After acclimatization for seven days, the rats were randomly and equally divided into five groups ( $\mathrm{n}=6$ for each group): control, model, COS, EVs, and EVs-COS. Except for the rats in the control group, the other rats were used to construct a cartilage injury model, as described previously [68]. Briefly, the rats were anesthetized by intraperitoneal injection of $3 \%$ pentobarbital sodium $(0.1 \mathrm{~mL} / 100 \mathrm{~g})$, and then the skin at the knee joint was incised. The joint capsule was cut from the medial edge of the patella, and
Table 1 The sequences of all primers

\begin{tabular}{|c|c|}
\hline Primer & Sequence $\left(5^{\prime}-3^{\prime}\right)$ \\
\hline GAPDH-ratF & AGACAGCCGCATCTTCTTGT \\
\hline GAPDH-ratR & CTTGCCGTGGGTAGAGTCAT \\
\hline IL-1 $\beta-r F$ & СCCTGCAGCTGGAGAGTGTGG \\
\hline $\mid L-1 \beta-r R$ & TGTGCTCTGCTTGAGAGGTGCT \\
\hline COL1A1-rF & GCATCAGGGTTTCAGAGCA \\
\hline COL1A1-rR & CGTTGGGTCATTTCCACAT \\
\hline COL2A1-rF & TTCCTCCGTCTACTGTCCACTGA \\
\hline COL2A1-rR & CTACATCATTGGAGCCCTGGAT \\
\hline OPN-rF & CCAGCCAAGGACCAACTACA \\
\hline OPN-rR & AGTGTTTGCTGTAATGCGCC \\
\hline ALP-rF & GCAGTATGAATTGAATCGGAACAAC \\
\hline ALP-rR & ATGGCCTGGTCCATCTCCAC \\
\hline c-Myc-rF & CTGCTGTCCTCCGAGTCCTC \\
\hline c-Myc-rR & GGGGGTTGCCTCTTTTCCAC \\
\hline OCN-rF & ACCATCTTTCTGCTCACTCTGCT \\
\hline OCN-rR & СCTTATTGCCCTCCTGCTTG \\
\hline$R \cup N \times 2-r F$ & CCACAGAGCTATTAAAGTGA \\
\hline RUNX2-rR & AACAAACTAGGTTTAGAGTCATCAAGC \\
\hline Akt1-rF & CTTTATTGGCTACAAGGAACGG \\
\hline Akt1-rR & CAGTCTGAATGGCGGTGGT \\
\hline PI3K-rF & ACGGCAATGTGGAGCAGA \\
\hline PI3K-rR & GTCGTAGCCAATCAGGGAG \\
\hline $\mathrm{BCl} 2-\mathrm{rF}$ & GATTGTGGCCTTCTTTGAGTTC \\
\hline $\mathrm{BCl} 2-\mathrm{rR}$ & CAGATGCCGGTTCAGGTACT \\
\hline p53-rF & GCTGAGTATCTGGACGACAGG \\
\hline p53-rR & AGCGTGATGATGGTAAGGATG \\
\hline Vps13a-rF & GCATTGAGAATACCGTTTG \\
\hline Vps13a-rR & GAATCCCATCTGCTTTGT \\
\hline Birc6-rF & CACTCTGGGTCTACTGCT \\
\hline Birc6-rR & ATTAGGGTGACATGAAATAC \\
\hline Itga1-rF & CATCCCTCATAACACCACC \\
\hline |tga1-rR & GTTCCCATCCTCCATCTT \\
\hline Ifi2712b-rF & GGAGGAGTTGTGGCTGTG \\
\hline Ifi2712b-rR & AAATCTTGCATTGGAGGC \\
\hline Gli1-rF & СTCCCTCGTGGCTTTCAT \\
\hline Gli1-rR & GCGGCAGTCCGTCTCATA \\
\hline
\end{tabular}

the articular cartilage on the patellofemoral joint surface was exposed. Subsequently, a full-thickness cartilage defect model with a diameter of $1 \mathrm{~mm}$ and a depth of $0.2 \mathrm{~mm}$ was prepared at $2 \mathrm{~mm}$ above the intercondylar fossa. After modeling, the articular cavity was rinsed, and the joint capsule and skin incisions were sutured. After the operation, penicillin $(30,000 \mathrm{U} /$ time $)$ was injected intramuscularly once a day for three consecutive days. Two weeks later, the rats in the COS, EVs, and EVs-COS groups were injected with COS $(2 \mathrm{mg}), \mathrm{EVs}(100 \mu \mathrm{g})$, and EVs-COS $(100 \mu \mathrm{g})$, respectively, in the articular cavity 
once a week for 8 weeks. Rats in the control group were not treated.

After the experiments, cartilage tissues were obtained from the different groups. A portion of the tissues was used for hematoxylin-eosin (HE) staining and type I and type II collagen immunohistochemistry staining [69], while the rest of the model, the COS and EVs-COS groups, were sent to Yanzai Biotechnology (Shanghai) Co., Ltd (China) for RNA sequencing. In addition, five genes were chosen for RT-qPCR to further verify the reliability of the sequencing data. All animal experiments were conducted in accordance with the National Medical Advisory Committee (NMAC) guidelines and approved by the Ethics Committee of China Medical University (CMU2021187).

\section{Analysis of sequencing data}

The samples were divided into three comparison groups: COS vs. model, EVs-COS vs. model, and EVs-COS vs. COS. Differentially expressed genes (DEGs) between COS and model, EVs-COS and model, as well as EVsCOS and COS were screened using Limma package version 3.34.0 in R3.6.1 (https://bioconductor.org/packages/ release/bioc/html/limma.html) [70]. The threshold values for DEG selection were false discovery rate (FDR) $<0.05$ and $\mid \log _{2}$ fold change (FC) $\mid>1$. After that, Mfuzz version 2.42.0 (http://www.bioconductor.org/packages/release/ bioc/html/Mfuzz.html) [71] in R3.6.1 was used to analyze the expression patterns of the obtained DEGs, and the gene clustering of expression modules was obtained. Finally, the DEGs in each cluster were submitted for biological process (BP) of gene ontology (GO) and Kyoto Encyclopedia of Genes and Genomes (KEGG) pathway enrichment analyses using DAVID version 6.8 (https:// david.ncifcrf.gov/) [72,73]. Statistical significance was set at $P<0.05$.

\section{Statistical analysis}

All experiments were performed at least thrice, and data are expressed as the mean \pm standard deviation (SD). GraphPad Prism 5 (San Diego, CA, USA) was used for the statistical analyses. For multiple comparisons, a oneway analysis of variance (ANOVA) with a post-hoc Tukey test was applied. Differences were considered statistically significant at $\mathrm{P}<0.05$.

\footnotetext{
Abbreviations

COS: Chitosan oligosaccharides; EVs: Extracellular vesicles; AMSCs: Adipose mesenchymal stem cells; OA: Osteoarthritis; EVs-COS: EVs-COS conjugates; TEM: Transmission electron microscopy; NTA: Nanosight NS300 particle size analyzer; PI: Propidium iodide; NMAC: National Medical Advisory Committee; IL-1 $\beta$ : Interleukin-1 $\beta$; COL1A1: Collagen type I alpha 1 chain; COL2A1: Collagen type II alpha 1 chain; OPN: Secreted phosphoprotein 1; ALP: Alkaline phosphatase; OCN: Osteocalcin; RUNX2: RUNX family transcription factor 2;
}

c-Myc: Transcriptional regulator Myc-like; p53: Tumor protein p53; Bcl2: BCL2 apoptosis regulator; Akt1: AKT serine/threonine kinase 1; PI3K: Phosphatidylinositol 3-kinase, putative; SFRP5: Secreted frizzled related protein 5; SOX9: SRY-box transcription factor 9; GLI1: LI family zinc finger 1; COL3A1: Collagen type III alpha 1 chain; ITGA4: Integrin subunit alpha 4; BAD: BCL2 associated agonist of cell death; FOXO3: Forkhead box O3; PPP2R3A: Protein phosphatase 2 regulatory subunit $B^{\prime \prime}$ alpha; CPT1B: Carnitine palmitoyltransferase 1B; $A C A C B$ : Acetyl-CoA carboxylase beta; CACNA1S: Calcium voltage-gated channel subunit alpha1 S; CACNG1: Calcium voltage-gated channel auxiliary subunit gamma 1; MAPK12: Mitogen-activated protein kinase 12.

\section{Supplementary Information}

The online version contains supplementary material available at https://doi. org/10.1186/s12951-021-01086-x.

Additional file 1: Figure S1. Screen of the optimal concentrations of extracellular vesicles (EVs) and chitosan oligosaccharide (COS). A The cell viability of chondrocytes with different concentrations of EVs. ${ }^{* *} P<0.01$, compared with $0 \mu \mathrm{g} / \mathrm{mL}$ EVs. B The cell viability of chondrocytes with different concentrations of COS. *: $P<0.05$, compared with $0 \mu \mathrm{g} / \mathrm{mL} \operatorname{COS}$; **P $<0.05$, compared with $0 \mu \mathrm{g} / \mathrm{mL} \operatorname{COS}$.

Additional file 2: Figure S2. The identification of rat chondrocytes extracted from rats' cartilage tissues by type II collagen immunohistochemical staining.

Additional file 3: Figure S3. The differentially expressed genes (DEGs) between COS and model groups, between EVs-COS and model groups, as well as between EVs-COS and COS groups.

Additional file 4: Table S1. The differentially expressed genes (DEGs) in at least two comparison groups.

Additional file 5: Table S2. DEGs in each cluster used for biological process and Kyoto Encyclopedia of Genes and Genomes (KEGG) enrichment analyses.

\section{Acknowledgements}

This work was supported by the Liaoning Cancer Hospital and Institute (Shenyang) and China Medical University (Shenyang).

\section{Authors' contributions}

ShL performed the experiments and generated the data. JL and ShL made substantial contributions to the conception and design of the present study. WJ and SiL conducted data analysis and interpretation of the data. ShL and XW contributed to the drafting and revision of the manuscript. All authors revised the manuscript and agreed to be accountable for all aspects of the research to ensure that the accuracy or integrity of any part of the work are appropriately investigated and resolved. All authors read and approved the final manuscript.

\section{Funding}

The National Natural Science Foundation of China (NSFC) (81571832), the Key Research \& Development Project of Liaoning Province (2018225082), and the 2018 Scientist Partners of China Medical University (CMU) and Shenyang Branch of the Chinese Academy of Sciences (CAS) (HZHB2018013).

\section{Availability of data and materials}

The data that support the findings of this study are available from the corresponding author upon reasonable request.

\section{Declarations}

Ethics approval and consent to participate

The animal study was reviewed and approved by the Animal Care and Use Committee of the China Medical University (CMU2021187). 


\section{Consent for publication}

The founding sponsors had no role in the design of the study; in the collection, analyses, or interpretation of data; in the writing of the manuscript; and in the decision to publish the results.

\section{Competing interests}

The authors declare no conflict of interest.

\section{Author details}

'Department of Tissue Engineering, Center of 3D Printing \& Organ Manufacturing, School of Intelligent Medicine, China Medical University (CMU), No. 77 Puhe Road, Shenyang North New Area, Shenyang 110122, China. ${ }^{2}$ Department of Bone and Soft Tissue Tumor Surgery, Cancer Hospital of China Medical University, Liaoning Cancer Hospital \& Institute, Shenyang 110042, Liaoning, China. ${ }^{3}$ Department of Prosthodontics, School and Hospital of Stomatology, China Medical University, Liaoning Provincial Key Laboratory of Oral Diseases, Shenyang 110002, China. ${ }^{4}$ Center of Organ Manufacturing, Department of Mechanical Engineering, Tsinghua University, Beijing 100084, China.

\section{Received: 24 July 2021 Accepted: 13 October 2021}

Published online: 26 October 2021

\section{References}

1. Ojanen SP, Finnila MAJ, Makela JTA, Saarela K, Happonen E, Herzog W, Saarakkala S, Korhonen RK. Anterior cruciate ligament transection of rabbits alters composition, structure and biomechanics of articular cartilage and chondrocyte deformation 2 weeks post-surgery in a site-specific manner. J Biomech. 2020;98:109450.

2. Murphy MP, Koepke LS, Lopez MT, Tong X, Ambrosi TH, Gulati GS, Marecic O, Wang Y, Ransom RC, Hoover MY, et al. Articular cartilage regeneration by activated skeletal stem cells. Nat Med. 2020;26:1583-92.

3. Thomas AC, Hubbard-Turner T, Wikstrom EA, Palmieri-Smith RM. Epidemiology of posttraumatic osteoarthritis. J Athl Train. 2017;52:491-6.

4. Mandl LA. Osteoarthritis year in review 2018: clinical. Osteoarthritis Cartilage. 2019;27:359-64

5. Felson DT. Developments in the clinical understanding of osteoarthritis. Arthritis Res Ther. 2009;11:203.

6. Mobasheri A. The future of osteoarthritis therapeutics: targeted pharmacological therapy. Curr Rheumatol Rep. 2013;15:364.

7. Harirforoosh S, Asghar W, Jamali F. Adverse effects of nonsteroidal antiinflammatory drugs: an update of gastrointestinal, cardiovascular and renal complications. J Pharm Pharm Sci. 2013;16:821-47.

8. Bramhill J, Ross S, Ross G. Bioactive nanocomposites for tissue repair and regeneration: a review. Int J Environ Res Public Health. 2017;14:66.

9. Bertrand N, Leroux JC. The journey of a drug-carrier in the body: an anatomo-physiological perspective. J Control Release. 2012;161:152-63.

10. Zhang R, Ma J, Han J, Zhang W, Ma J. Mesenchymal stem cell related therapies for cartilage lesions and osteoarthritis. Am J Transl Res. 2019;11:6275-89.

11. Fu X, Liu G, Halim A, Ju Y, Luo Q, Song AG. Mesenchymal stem cell migration and tissue repair. Cells. 2019;8:784.

12. Doyle EC, Wragg NM, Wilson SL. Intraarticular injection of bone marrowderived mesenchymal stem cells enhances regeneration in knee osteoarthritis. Knee Surg Sports Traumatol Arthrosc. 2020;28:3827-42.

13. Mirsaidi A, Kleinhans KN, Rimann M, Tiaden AN, Stauber M, Rudolph KL, Richards PJ. Telomere length, telomerase activity and osteogenic differentiation are maintained in adipose-derived stromal cells from senile osteoporotic SAMP6 mice. J Tissue Eng Regen Med. 2012;6:378-90.

14. Lee WS, Kim HJ, Kim Kl, Kim GB, Jin W. Intra-articular injection of autologous adipose tissue-derived mesenchymal stem cells for the treatment of knee osteoarthritis: a phase $\mathrm{lb}$, randomized, placebo-controlled clinical trial. Stem Cells Transl Med. 2019;8:504-11.

15. Liu X, Du M, Wang Y, Liu S, Liu X. BMP9 overexpressing adipose-derived mesenchymal stem cells promote cartilage repair in osteoarthritisaffected knee joint via the Notch1/Jagged1 signaling pathway. Exp Ther Med. 2018;16:4623-31.

16. van Niel G, D'Angelo G, Raposo G. Shedding light on the cell biology of extracellular vesicles. Nat Rev Mol Cell Biol. 2018;19:213-28.
17. Li X, Corbett AL, Taatizadeh E, Tasnim N, Little JP, Garnis C, Daugaard M, Guns E, Hoorfar M, Li ITS. Challenges and opportunities in exosome research-perspectives from biology, engineering, and cancer therapy. APL Bioeng. 2019;3:011503

18. Fatima F, Nawaz M. Stem cell-derived exosomes: roles in stromal remodeling, tumor progression, and cancer immunotherapy. Chin J Cancer. 2015;34:541-53.

19. Hong $\mathrm{P}$, Yang H, Wu Y, Li K, Tang Z. The functions and clinical application potential of exosomes derived from adipose mesenchymal stem cells: a comprehensive review. Stem Cell Res Ther. 2019;10:242.

20. Cao G, Chen B, Zhang X, Chen H. Human adipose-derived mesenchymal stem cells-derived exosomal microRNA-19b promotes the healing of skin wounds through modulation of the CCL1/TGF-beta signaling axis. Clin Cosmet Investig Dermatol. 2020;13:957-71.

21. Zhao C, Chen JY, Peng WM, Yuan B, Bi Q, Xu YJ. Exosomes from adiposederived stem cells promote chondrogenesis and suppress inflammation by upregulating miR145 and miR221. Mol Med Rep. 2020;21:1881-9.

22. Wang W, Meng Q, Li Q, Liu J, Zhou M, Jin Z, Zhao K. Chitosan derivatives and their application in biomedicine. Int J Mol Sci. 2020;21:487.

23. Wang $X, M a ~ J$, Wang $Y$, He B. Structural characterization of phosphorylated chitosan and their applications as effective additives of calcium phosphate cements. Biomaterials. 2001;22:2247-55.

24. Wang X, Ma J, Feng Q, Cui F. Skeletal repair in rabbits with calcium phosphate cements incorporated phosphorylated chitin. Biomaterials. 2002;23:4591-600.

25. Wang $X$, Ma J, Wang $Y$, He $B$. Bone repair in radii and tibias of rabbits with phosphorylated chitosan reinforced calcium phosphate cements. Biomaterials. 2002;23:4167-76.

26. Zhao X, Zhou L, Li Q, Zou Q, Du C. Biomimetic mineralization of carboxymethyl chitosan nanofibers with improved osteogenic activity in vitro and in vivo. Carbohydr Polym. 2018;195:225-34.

27. Li W, Liu Y, Zhang P, Tang Y, Zhou M, Jiang W, Zhang X, Wu G, Zhou $Y$. Tissue-engineered bone immobilized with human adipose stem cells-derived exosomes promotes bone regeneration. ACS Appl Mater Interfaces. 2018;10:5240-54.

28. Park ST, Kim J. Trends in next-generation sequencing and a new era for whole genome sequencing. int neurourol j. 2016;20:s76-83.

29. Soares Martins T, Catita J, Martins Rosa I. O ABdCES, Henriques AG: exosome isolation from distinct biofluids using precipitation and columnbased approaches. PLoS One. 2018;13:e0198820.

30. Mianehsaz E, Mirzaei HR, Mahjoubin-Tehran M, Rezaee A, Sahebnasagh R, Pourhanifeh MH, Mirzaei H, Hamblin MR. Mesenchymal stem cell-derived exosomes: a new therapeutic approach to osteoarthritis? Stem Cell Res Ther. 2019;10:340.

31. Zhang C, Liao Q, Ming JH, Hu GL, Chen Q, Liu SQ, Li YM. The effects of chitosan oligosaccharides on OPG and RANKL expression in a rat osteoarthritis model. Acta Cir Bras. 2017:32:418-28.

32. Liu Y, Lin L, Zou R, Wen C, Wang Z, Lin F. MSC-derived exosomes promote proliferation and inhibit apoptosis of chondrocytes via IncRNA-KLF3-AS1/ miR-206/GIT1 axis in osteoarthritis. Cell Cycle. 2018;17:2411-22.

33. Wang J, Wang $X$, Cao Y, Huang T, Song DX, Tao HR. Therapeutic potential of hyaluronic acid/chitosan nanoparticles for the delivery of curcuminoid in knee osteoarthritis and an in vitro evaluation in chondrocytes. Int J Mol Med. 2018:42:2604-14.

34. Wang BW, Jiang Y, Yao ZL, Chen PS, Yu B, Wang SN. Aucubin protects chondrocytes against IL-1 beta-Induced apoptosis in vitro and inhibits osteoarthritis in mice model. Drug Des Devel Ther. 2019;13:3529-38.

35. Kuivaniemi H, Tromp G. Type III collagen (COL3A1): gene and protein structure, tissue distribution, and associated diseases. Gene. 2019:707:151-71.

36. Jain E, Chinzei N, Blanco A, Case N, Sandell L, Sell S, Rai MF, Zustiak SP. Platelet-rich plasma released from polyethylene glycol hydrogels exerts beneficial effects on human chondrocytes. J Orthop Res. 2019;37:2401-10

37. Yin N, Zhu L, Ding L, Yuan J, Du L, Pan M, Xue F, Xiao H. MiR-135-5p promotes osteoblast differentiation by targeting HIF1AN in MC3T3-E1 cells. Cell Mol Biol Lett. 2019;24:51.

38. Ma J, Wang Z, Zhao J, Miao W, Ye T, Chen A. Resveratrol attenuates lipopolysaccharides (LPS)-induced inhibition of osteoblast differentiation in MC3T3-E1 cells. Med Sci Monit. 2018;24:2045-52. 
39. Wakasugi T, Shimizu I, Yoshida Y, Hayashi Y, Ikegami R, Suda M, Katsuumi G, Nakao M, Hoyano M, Kashimura T, et al. Role of smooth muscle cell p53 in pulmonary arterial hypertension. PLoS ONE. 2019;14:e0212889.

40. Nebbioso A, Carafa V, Conte M, Tambaro FP, Abbondanza C, Martens J, Nees M, Benedetti R, Pallavicini I, Minucci S, et al. c-Myc modulation and acetylation is a key HDAC inhibitor target in cancer. Clin Cancer Res. 2017;23:2542-55.

41. Iannone F, De Bari C, Scioscia C, Patella V, Lapadula G. Increased BCl-2/ p53 ratio in human osteoarthritic cartilage: a possible role in regulation of chondrocyte metabolism. Ann Rheum Dis. 2005;64:217-21.

42. Mei S, Ge S, Wang J, Li H, Jing X, Liang K, Zhang X, Xue C, Zhang C, Zhang T. PRMT5 promotes progression of endometrioid adenocarcinoma via ERalpha and cell cycle signaling pathways. J Pathol Clin Res. 2021;7:154-64.

43. Liu R, Chen Y, Liu G, Li C, Song Y, Cao Z, Li W, Hu J, Lu C, Liu Y. PI3KJAKT pathway as a key link modulates the multidrug resistance of cancers. Cell Death Dis. 2020;11:797.

44. Xu K, Sha Y, Wang S, Chi Q, Liu Y, Wang C, Yang L. Effects of Bakuchiol on chondrocyte proliferation via the PI3K-Akt and ERK1/2 pathways mediated by the estrogen receptor for promotion of the regeneration of knee articular cartilage defects. Cell Prolif. 2019;52:e12666.

45. Maeda K, Kobayashi Y, Koide M, Uehara S, Okamoto M, Ishihara A, Kayama T, Saito M, Marumo K. The regulation of bone metabolism and disorders by Wnt signaling. Int J Mol Sci. 2019;20:5525.

46. Lietman C, Wu B, Lechner S, Shinar A, Sehgal M, Rossomacha E, Datta P, Sharma A, Gandhi R, Kapoor M, Young PP. Inhibition of Wnt/beta-catenin signaling ameliorates osteoarthritis in a murine model of experimental osteoarthritis. JCI Insight. 2018;3:e96308.

47. Carling D. AMPK signalling in health and disease. Curr Opin Cell Biol. 2017;45:31-7.

48. Wang J, Fang L, Ye L, Ma S, Huang H, Lan X, Ma J. miR-137 targets the inhibition of TCF4 to reverse the progression of osteoarthritis through the AMPK/NF-kappaB signaling pathway. Biosci Rep. 2020;40:BSR20200466.

49. Chen Y, Shou K, Gong C, Yang H, Yang Y, Bao T. Anti-inflammatory effect of geniposide on osteoarthritis by suppressing the activation of p38 MAPK signaling pathway. Biomed Res Int. 2018;2018:8384576.

50. Chen H, He Y, Wu D, Dai G, Zhao C, Huang W, Jiang D. Bone marrow sFRP5 level is negatively associated with bone formation markers. Osteoporos Int. 2017;28:1305-11.

51. Lefebvre V, Angelozzi M, Haseeb A. SOX9 in cartilage development and disease. Curr Opin Cell Biol. 2019;61:39-47.

52. Shi Y, He G, Lee WC, McKenzie JA, Silva MJ, Long F. Gli1 identifies osteogenic progenitors for bone formation and fracture repair. Nat Commun. 2017:8:2043.

53. Zhang Y, Yang Y, Wang C, Wan S, Yao Z, Zhang Y, Liu J, Zhang C. Identification of diagnostic biomarkers of osteoarthritis based on multi-chip integrated analysis and machine learning. DNA Cell Biol. 2020. https://doi. org/10.1089/dna.2020.5552.

54. Lee Kl, Choi S, Matsuzaki T, Alvarez-Garcia O, Olmer M, Grogan SP, D'Lima DD, Lotz MK. FOXO1 and FOXO3 transcription factors have unique functions in meniscus development and homeostasis during aging and osteoarthritis. Proc Natl Acad Sci USA. 2020;117:3135-43.

55. Attia HR, Ibrahim MH, El-Aziz SHA, Hassan NM, Osman RA, Hagag HA, Yassa ME, Abdelrahman AH, Salama II, Sobeih ME. ITGA4 gene methylation status in chronic lymphocytic leukemia. Future Sci OA. 2020;6:FSO583.

56. Pulkka OP, Mpindi JP, Tynninen O, Nilsson B, Kallioniemi O, Sihto $\mathrm{H}$, Joensuu $\mathrm{H}$. Clinical relevance of integrin alpha 4 in gastrointestinal stromal tumours. J Cell Mol Med. 2018;22:2220-30.
57. Zhang X, Wan S, Yu Y, Ruan W, Wang H, Xu L, Wang C, Chen S, Cao T, Peng $Q$, et al. Identifying potential DNA methylation markers in early-stage colorectal cancer. Genomics. 2020;112:3365-73.

58. Li J, Zhang L, Zheng Y, Shao R, Liang Q, Yu W, Wang H, Zou W, Wang D, Xiang J, Lin A. BAD inactivation exacerbates rheumatoid arthritis pathology by promoting survival of sublining macrophages. Elife. 2020;9:e56309.

59. Dzulko M, Pons M, Henke A, Schneider G, Kramer OH. The PP2A subunit PR130 is a key regulator of cell development and oncogenic transformation. Biochim Biophys Acta Rev Cancer. 2020;1874:188453.

60. Chen H, Xu J, Wang P, Shu Q, Huang L, Guo J, Zhang X, Zhang H, Wang $Y$, Shen $Z$, et al. Protein phosphatase 2 regulatory subunit B"Alpha silencing inhibits tumor cell proliferation in liver cancer. Cancer Med. 2019;8:7741-53.

61. Tzanavari T, Varela A, Theocharis S, Ninou E, Kapelouzou A, Cokkinos DV, Kontaridis MI, Karalis KP. Metformin protects against infection-induced myocardial dysfunction. Metabolism. 2016;65:1447-58.

62. Kim JM, Heo HS, Shin SC, Kwon HK, Lee JC, Sung ES, Kim HS, Park GC, Lee BJ. Increased calcium channel in the lamina propria of aging rat. Aging. 2019;11:8810-24

63. Lin J, Yang J, Xu X, Wang Y, Yu M, Zhu Y. A robust 11-genes prognostic model can predict overall survival in bladder cancer patients based on five cohorts. Cancer Cell Int. 2020;20:402.

64. Thery C, Amigorena S, Raposo G, Clayton A. Isolation and characterization of exosomes from cell culture supernatants and biological fluids. Curr Protoc Cell Biol. 2006;30:3-22.

65. Lee YS, Kim SY, Ko E, Lee JH, Yi HS, Yoo YJ, Je J, Suh SJ, Jung YK, Kim JH, et al. Exosomes derived from palmitic acid-treated hepatocytes induce fibrotic activation of hepatic stellate cells. Sci Rep. 2017;7:3710.

66. Wu M, Ouyang Y, Wang Z, Zhang R, Huang PH, Chen C, Li H, Li P, Quinn $\mathrm{D}$, Dao $\mathrm{M}$, et al. Isolation of exosomes from whole blood by integrating acoustics and microfluidics. Proc Natl Acad Sci U S A. 2017;114:10584-9.

67. Zhou Q, Yang H, Pan H, Pan H, Zhou J. Exosomes isolated from the miR215-modified bone marrow mesenchymal stem cells protect $\mathrm{H}_{2} \mathrm{O} 2-$ induced rat myoblasts via the miR-215/FABP3 pathway. Exp Mol Pathol. 2021;119:104608.

68. Chan DD, Li J, Luo W, Predescu DN, Cole BJ, Plaas A. Pirfenidone reduces subchondral bone loss and fibrosis after murine knee cartilage injury. J Orthop Res. 2018;36:365-76.

69. Zu Y, Mu Y, Li Q, Zhang ST, Yan HJ. Icariin alleviates osteoarthritis by inhibiting NLRP3-mediated pyroptosis. J Orthop Surg Res. 2019;14:307.

70. Ritchie ME, Phipson B, Wu D, Hu Y, Law CW, Shi W, Smyth GK. limma powers differential expression analyses for RNA-sequencing and microarray studies. Nucleic Acids Res. 2015;43:e47.

71. Kumar L, Futschik ME. Mfuzz: a software package for soft clustering of microarray data. Bioinformation. 2007;2:5-7.

72. da Huang W, Sherman BT, Lempicki RA. Systematic and integrative analysis of large gene lists using DAVID bioinformatics resources. Nat Protoc. 2009:4:44-57.

73. da Huang W, Sherman BT, Lempicki RA. Bioinformatics enrichment tools: paths toward the comprehensive functional analysis of large gene lists. Nucleic Acids Res. 2009;37:1-13.

\section{Publisher's Note}

Springer Nature remains neutral with regard to jurisdictional claims in published maps and institutional affiliations. 